\title{
Terpenoids and Their Biological Activities from Cinnamomum: A Review
}

\author{
Meiting Wu, ${ }^{1}$ Lin Ni $\mathbb{D}^{1,2}$ Haixiao Lu, ${ }^{3}$ Huiyou Xu ${ }^{1},{ }^{1}$ Shuangquan Zou $\mathbb{D}^{2}$, \\ and Xiaoxing $\mathrm{Zou}^{2}$ \\ ${ }^{1}$ College of Plant Protection, Fujian Agriculture and Forestry University, Fuzhou 350002, China \\ ${ }^{2}$ Fujian Colleges and Universities Engineering Research Institute of Conservation \& Utilization of Natural Bioresources, \\ Fujian Agriculture and Forestry University, Fuzhou 350002, China \\ ${ }^{3}$ Yulin Normal University, YuLin 537000, China \\ Correspondence should be addressed to Lin Ni; nilin_fjau@126.com
}

Received 28 April 2020; Revised 4 June 2020; Accepted 15 June 2020; Published 14 July 2020

Academic Editor: Ponnurengam Malliappan Sivakumar

Copyright ( 2020 Meiting Wu et al. This is an open access article distributed under the Creative Commons Attribution License, which permits unrestricted use, distribution, and reproduction in any medium, provided the original work is properly cited.

Cinnamomum is a genus of the family Lauraceae, which has been recognized worldwide as an important genus due to its beneficial uses. A great deal of research on its phytochemistry and pharmacological effects has been conducted. It is noteworthy that terpenoids are the characteristic of Cinnamomum due to the peculiar structures and significant biological effects. For a more indepth study and the better use of Cinnamomum plants in the future, the chemical structures and biological effects of terpenoids obtained from Cinnamomum were summarized in the present study. To date, a total of 181 terpenoids with various skeletons have been isolated from Cinnamomum. These compounds have been demonstrated to play an important role in immunomodulatory, anti-inflammatory, antimicrobial, antioxidant, and anticancer activities. However, studies on the bioactive components from Cinnamomum plants have only focused on a dozen species. Hence, further studies on the potential pharmacological effects need to be conducted in the future.

\section{Introduction}

Cinnamomum, which is a genus of the family Lauraceae, is represented by evergreen trees and shrubs [1]. Approximately 250 species of this genus have been found around the world and are mainly distributed in tropical and subtropical regions of Southeast Asia, Australia, and North, Central, and South America. There are approximately 46 species in China, and these are mainly in the southern religions. Yunnan province has the most species, followed by Guangdong and Sichuan [2, 3].

Cinnamomum has been recognized worldwide as an important genus due to its beneficial uses. This has been traditionally used in flavoring food and in folk medicine for its sweating, antipyretic, and analgesic effects [4]. Some Cinnamomum species, including C. cassia, C. zeylanicum, C. tamala, and C. wilsonii, are famous herbs that have a long history of being used as medicine. In addition, the extracted essential oil from C. camphora, with the main ingredients of eucalyptol, linalool, and camphor, is an important raw material for the chemical industry and medicine. Furthermore, the Cinnamomi cortex, which is obtained from some Cinnamomum species, has been used for treating cardiovascular, chronic gastrointestinal, and inflammatory diseases $[5,6]$. Cinnamaldehyde, which is the main constituent of the volatile oil of $C$. cassia bark, has been mainly used in medicine, foods, and cosmetics and has been proven to exert antifungal and antibacterial activities [7].

To date, many studies on phytochemistry of the genus Cinnamomum have been conducted. Over 500 compounds have been obtained from Cinnamomum plants, which cover lignans, terpenoids, flavonoids, phenylpropanoids, alkaloids, steroids, and butanolides. Among these compounds, approximately 300 compounds are from C. Cassia, which is the most thoroughly studied. This has been reported to show many special contents, particularly terpenoids. Other species 
also contain a number of unique terpenoids with rare structures, which can only be found from certain species. More importantly, terpenoids, which are the most abundant and diverse compounds in this genus, have also been proven to show great pharmacological activities, including immunomodulatory, anti-inflammatory, antimicrobial, antioxidant, and anticancer activities. Thus, the present review aimed at summarizing the phytochemistry and pharmacological effects of terpenoids from Cinnamomum species and providing a basis for the future in-depth study of the genus Cinnamomum.

\section{Chemical Constituents and Biological Activities}

A number of studies on the phytochemistry of the genus Cinnamomum have been conducted, and focus has been given on approximately 14 species. From these studies, the genus has been shown to possess abundant and various terpenoids with unique structures. To date, a total of 181 terpenoids have been isolated from the genus Cinnamomum, including 43 monoterpenes, 83 sesquiterpenes, 53 diterpenes, and two triterpenes. Among these compounds, 119 terpenoids were obtained from C. cassia, 21 terpenoids were obtained from $C$. wilsonii, 19 terpenoids were obtained from C. camphora, 18 terpenoids were obtained from C. glanduliferum, 17 terpenoids were obtained from C. subavenium, eight terpenoids were obtained from $C$. zeylanicum, eight terpenoids were obtained from C. osmophloeum, three terpenoids were obtained from $C$. inunctum, three terpenoids were obtained from $C$. philippinense, two terpenoids were obtained from $C$. kotoense, one terpenoid was obtained from C. burmannii, one terpenoid was obtained from C. parthenoxylon, one terpenoid was obtained from C. reticulatum, and one terpenoid was obtained from C. tenuifolium, respectively.

2.1. Monoterpenes. A total of 43 monoterpenes have been reported from Cinnamomum (Table 1 and Figure 1), including 24 cyclic monoterpenes (1-17, 37-43) and 19 acyclic monoterpenes (18-36). Among these cyclic monoterpenes, compounds 1-13 are menthane monoterpenes, which are the most abundant types in the genus Cinnamomum. In addition, compounds $\mathbf{6}-\mathbf{- 1 2}$ are the common constituents in the volatile oils of Cinnamomum plants. Compounds 14 and 15 are camphane monoterpenes and camphor (15), along with linalool (29) and eucalyptol (41), is one of the main components of volatile oil in C. camphora. Compounds $\mathbf{1 6}$ and $\mathbf{1 7}$ belong to the pinane-type monoterpenes, which are common in volatile oils. Monoterpenes 18-36 are lauranetype monoterpenes. Among these, compounds 18-24 have a geranylphenylacetate skeleton connected to glucose. Compounds 18-21, 23, and 24 were further cyclized to form a tetrahydrofuran ring. Interestingly, these compounds were only isolated from $C$. cassia and are the characteristic compounds for this species. Extracted from C. inunctum, compounds 25-27 were monoterpene lactones. Based on this structure, the original hydroxyl groups at the C-4 and C-7 positions of compound 27 were further cyclized to form a rare spironolactone structure. Obtained from C. camphora, compound $\mathbf{3 3}$ is an acyclic monoterpene with one carbon degraded and has shown strong anti-inflammatory activity. Compounds 37 and 38 are normonoterpenes from $C$. reticulatum, with one carbon degraded. Subamone (43), which was obtained from $C$. subavenium, has a cycloheptanone skeleton and has strong antitumor activity.

Some monoterpenes from Cinnamomum plants have shown potent antimicrobial activities. Carvacrol (4) showed a broad spectrum of antibacterial activity against both Grampositive and Gram-negative bacteria [25]. The antimicrobial activity of compound $\mathbf{4}$ against numerous bacteria was studied and it showed a high inhibitory effect against all these strains, including Staphylococcus aureus, Staphylococcus epidermidis, Streptococcus pneumonia, Escherichia coli, Klebsiella pneumonia, Enterobacter spp., Serratia spp., and Proteus mirabilis [26]. Furthermore, 4 is also effective against various fungi, such as Aspergillus niger, Aspergillus flavus, Alternaria alternata, Penicillium rubrum, Trichoderma viride, Candida spp., and dermatophytes [27]. The antibacterial activity of compounds 4 and 9 (a precursor of 4 ) was studied against the foodborne microorganism $V$. cholerae. Compound 4 exerted a good inhibitory effect against $V$. cholerae, while $\mathbf{9}$ exhibited a weak activity. However, it was interesting to find that the inhibitory effects were enhanced when the two compounds were used together, showing a synergistic effect [28]. According to the findings both 16 and 29 exerted antibacterial activity against E. faecalis and $S$. aureus, and $\mathbf{1 6}$ showed antifungal activity against C. albicans. Similarly, compound 7 was effective against Ae. aegypti and Ae. albopictus [29], as well as Culex pipiens molestus [30].

The antioxidant activities of some monoterpenes were evaluated. The assessment on antioxidant capacity of carvacrol (4) showed that 4 at the doses of $25(49.79 \mathrm{IU} / \mathrm{mg})$ and $50 \mathrm{mg} / \mathrm{kg}$ (52.43 IU/mg) enhanced the level of hippocampal SOD activity in ischemic rats, when compared with the ischemic group $(42.9 \mathrm{IU} / \mathrm{mg})$. In the $\mathrm{DPPH}$ assay, the percentage of oxidant inhibition was significantly increased in the ischemic group treated with 4 at doses of 25 (41.5\%) and $50 \mathrm{mg} / \mathrm{kg}$ (36.83\%), when compared to the untreated ischemic group (11.29\%) [31]. Hakimi et al. confirmed that three doses of compound $4(25,50$, and $100 \mathrm{mg} / \mathrm{kg})$ could significantly increase the thiol content and improve the activities of both CAT and SOD compared with LPS groups in the hippocampus of rats [32]. In the trolox equivalent antioxidant capacity assay, the antioxidant effects of compounds $\mathbf{4}$ and $\mathbf{5}$ were evaluated and compared to that of the synthetic antioxidant trolox. Both compounds have exerted significant antioxidant activity similar to trolox, and the capacity of $\mathbf{4}$ has been proven to be higher than that of its isomer thymol at the same concentration [25]. Moreover, the antioxidant potential of p-cymene (9) was evaluated in the hippocampus of adult mice. The treatment with 9 at a concentration of $50 \mathrm{mg} / \mathrm{kg}(0.42 \pm 0.03)$ has produced a significant decrease in lipid peroxidation $65.54 \%$, when compared with the control group. Furthermore, compound 9 at all tested doses (50, 100, and $150 \mathrm{mg} / \mathrm{kg}$ ) could significantly decrease the nitrite content and produce an increase in SOD and catalase activities [33]. 
TABle 1: Monoterpenes from the genus Cinnamomum.

\begin{tabular}{|c|c|c|c|c|c|c|}
\hline No. & Type & Compound & $\begin{array}{l}\text { Molecular } \\
\text { formula }\end{array}$ & Molecular weight & Origin & References \\
\hline 1 & \multirow{13}{*}{ Menthane } & $\begin{array}{l}\text { (3R,4R)-p-Menth-1-ene-3,4-diol } \\
\text { 3-O- } \beta \text {-D-glucopyranoside }\end{array}$ & $\mathrm{C}_{16} \mathrm{H}_{28} \mathrm{O}_{7}$ & 332 & s & {$[8]$} \\
\hline 2 & & $\begin{array}{c}\text { (3R,4S,6R)-p-Menth-1-ene-3,6-diol } \\
\text { 3-O- } \beta \text {-D -glucopyranoside }\end{array}$ & $\mathrm{C}_{16} \mathrm{H}_{28} \mathrm{O}_{7}$ & 332 & $\mathrm{~s}$ & {$[8]$} \\
\hline 3 & & (4R)-p-Menthane-1,2 $\alpha, 8$-triol & $\mathrm{C}_{10} \mathrm{H}_{20} \mathrm{O}_{3}$ & 188 & s & {$[8]$} \\
\hline 4 & & Carvacrol & $\mathrm{C}_{10} \mathrm{H}_{14} \mathrm{O}$ & 150 & $\mathrm{~s}$ & [9] \\
\hline 5 & & Thymol & $\mathrm{C}_{10} \mathrm{H}_{14} \mathrm{O}$ & 150 & s & [9] \\
\hline 6 & & $\alpha$-Terpineol & $\mathrm{C}_{10} \mathrm{H}_{18} \mathrm{O}$ & 154 & $c, d, g, o$ & {$[10-12]$} \\
\hline 7 & & $\alpha$-Phellandrene & $\mathrm{C}_{10} \mathrm{H}_{16}$ & 136 & d, g & {$[10,13]$} \\
\hline 8 & & $\alpha$-Terpinene & $\mathrm{C}_{10} \mathrm{H}_{16}$ & 136 & $\mathrm{~d}, \mathrm{~g}$ & {$[10,13]$} \\
\hline 9 & & p-Cymene & $\mathrm{C}_{10} \mathrm{H}_{14}$ & 134 & g & {$[10]$} \\
\hline 10 & & Terpinolene & $\mathrm{C}_{10} \mathrm{H}_{16}$ & 136 & d, $g$ & {$[10,13]$} \\
\hline 11 & & Terpinen-4-ol & $\mathrm{C}_{10} \mathrm{H}_{18} \mathrm{O}$ & 154 & $\mathrm{~g}$ & {$[10]$} \\
\hline 12 & & Limonene & $\mathrm{C}_{10} \mathrm{H}_{16}$ & 136 & $\mathrm{~g}, \mathrm{z}$ & {$[10,11]$} \\
\hline 13 & & $\begin{array}{l}\text { (1R,2R,4S,6S)-4-(2-Hydroxypropan-2-yl) } \\
\text {-1-methyl-7-oxabicy clo[4.1.0]heptan-2-ol }\end{array}$ & $\mathrm{C}_{10} \mathrm{H}_{18} \mathrm{O}_{3}$ & 186 & c & {$[14]$} \\
\hline 14 & \multirow{2}{*}{ Camphane } & Borneol & $\mathrm{C}_{10} \mathrm{H}_{18} \mathrm{O}$ & 154 & $c, g, z, o$ & {$[10-12]$} \\
\hline 15 & & Camphor & $\mathrm{C}_{10} \mathrm{H}_{16} \mathrm{O}$ & 152 & $c, d, g$ & {$[10,13]$} \\
\hline 16 & \multirow{2}{*}{ Pinane } & $\alpha$-Pinene & $\mathrm{C}_{10} \mathrm{H}_{16}$ & 136 & $\mathrm{~d}, \mathrm{~g}, \mathrm{z}$ & {$[10,11]$} \\
\hline 17 & & $\beta$-Pinene & $\mathrm{C}_{10} \mathrm{H}_{16}$ & 136 & $c, d, g$ & {$[10,11]$} \\
\hline 18 & \multirow{19}{*}{ Laurane } & Cinnacasside A & $\mathrm{C}_{25} \mathrm{H}_{36} \mathrm{O}_{11}$ & 512 & $\mathrm{c}$ & {$[15-17]$} \\
\hline 19 & & Cinnacasside B & $\mathrm{C}_{25} \mathrm{H}_{36} \mathrm{O}_{11}$ & 512 & c & {$[15-17]$} \\
\hline 20 & & Cinnacasside $\mathrm{C}$ & $\mathrm{C}_{25} \mathrm{H}_{36} \mathrm{O}_{11}$ & 512 & c & {$[15-17]$} \\
\hline 21 & & Cinnacasside D & $\mathrm{C}_{25} \mathrm{H}_{36} \mathrm{O}_{11}$ & 512 & c & [15] \\
\hline 22 & & Cinnacasside E & $\mathrm{C}_{25} \mathrm{H}_{38} \mathrm{O}_{11}$ & 514 & c & {$[15]$} \\
\hline 23 & & Cinnacasside F & $\mathrm{C}_{26} \mathrm{H}_{40} \mathrm{O}_{12}$ & 544 & c & {$[17]$} \\
\hline 24 & & Cinnacasside $\mathrm{G}$ & $\mathrm{C}_{26} \mathrm{H}_{40} \mathrm{O}_{12}$ & 545 & c & {$[17]$} \\
\hline 25 & & $\begin{array}{c}\text { 5-(2,3-Dihydroxy-3-methylbutyl) } \\
\text {-4-hydroxy-4-methyldihydrofuran-2(3H)-one }\end{array}$ & $\mathrm{C}_{10} \mathrm{H}_{18} \mathrm{O}_{5}$ & 218 & $\mathrm{i}$ & {$[18]$} \\
\hline 26 & & $\begin{array}{l}\text { 5-(2,3-Dihydroxy-3-methylbutyl) } \\
\text {-4-methylfuran-2(5H)-one }\end{array}$ & $\mathrm{C}_{10} \mathrm{H}_{16} \mathrm{O}_{4}$ & 200 & $\mathrm{i}$ & {$[18]$} \\
\hline 27 & & $\begin{array}{l}\text { 8-Hydroxy-4,7,7-trimethyl } \\
\text {-9-1,6-dioxaspiro[4.4]non-3-en-2-one }\end{array}$ & $\mathrm{C}_{10} \mathrm{H}_{14} \mathrm{O}_{4}$ & 198 & $\mathrm{i}$ & {$[18]$} \\
\hline 28 & & $\begin{array}{l}\text { trans-Linalool-3,6-oxide } \\
\text { - } \beta \text {-D-glucopyranoside }\end{array}$ & $\mathrm{C}_{16} \mathrm{H}_{28} \mathrm{O}_{7}$ & 332 & c & {$[19]$} \\
\hline 29 & & Linalool & $\mathrm{C}_{10} \mathrm{H}_{18} \mathrm{O}$ & 154 & $c, d$ & {$[19,20]$} \\
\hline 30 & & $\begin{array}{c}\text { 3,7-Dimethyl-1-octene } \\
\text {-3,6,7-triol }\end{array}$ & $\mathrm{C}_{10} \mathrm{H}_{20} \mathrm{O}_{3}$ & 188 & c & {$[19]$} \\
\hline 31 & & $\begin{array}{l}\text { 3,7-Dimethyl-oct-1-en-3,6,7 } \\
\text {-triol-6-O- } \beta \text {-D-glucopyranoside }\end{array}$ & $\mathrm{C}_{16} \mathrm{H}_{30} \mathrm{O}_{8}$ & 350 & c & {$[19]$} \\
\hline 32 & & (6R)-Geraniol-6,7-diol & $\mathrm{C}_{10} \mathrm{H}_{20} \mathrm{O}_{3}$ & 188 & c & {$[19]$} \\
\hline 33 & & 6-Hydroxy-6-methyl-4,7-octadien-2-one & $\mathrm{C}_{9} \mathrm{H}_{14} \mathrm{O}_{2}$ & 154 & $\mathrm{~d}$ & {$[21]$} \\
\hline 34 & & $\beta$-Ocimene & $\mathrm{C}_{10} \mathrm{H}_{16}$ & 136 & $\mathrm{~d}$ & {$[13]$} \\
\hline 35 & & $\beta$-Myrcene & $\mathrm{C}_{10} \mathrm{H}_{16}$ & 136 & $\mathrm{~g}$ & {$[10]$} \\
\hline 36 & & Geranyl acetate & $\mathrm{C}_{12} \mathrm{H}_{20} \mathrm{O}_{2}$ & 196 & c & {$[20]$} \\
\hline 37 & \multirow[b]{2}{*}{ Normonoterpenes } & Reticuone & $\mathrm{C}_{9} \mathrm{H}_{14} \mathrm{O}_{3}$ & 170 & $\mathrm{r}$ & {$[22]$} \\
\hline 38 & & $\begin{array}{l}\text { (3,3-Dimethylcyclohex-1-ene-1,4-diyl) } \\
\text { dimethanol }\end{array}$ & $\mathrm{C}_{15} \mathrm{H}_{26} \mathrm{O}_{7}$ & 318 & c & {$[14]$} \\
\hline 39 & \multirow{5}{*}{ Others } & $\alpha$-Thujene & $\mathrm{C}_{10} \mathrm{H}_{16}$ & 136 & d, $g$ & {$[10]$} \\
\hline 40 & & Sabinene & $\mathrm{C}_{10} \mathrm{H}_{16}$ & 136 & $\mathrm{~d}, \mathrm{~g}$ & [10] \\
\hline 41 & & Eucalyptol & $\mathrm{C}_{10} \mathrm{H}_{18} \mathrm{O}$ & 154 & $\mathrm{~d}, \mathrm{~g}$ & {$[10,13]$} \\
\hline 42 & & Camphene & $\mathrm{C}_{10} \mathrm{H}_{16}$ & 136 & $c, d, g$ & {$[13,20]$} \\
\hline 43 & & Subamone & $\mathrm{C}_{10} \mathrm{H}_{16} \mathrm{O}_{2}$ & 168 & $\mathrm{~s}$ & {$[23,24]$} \\
\hline
\end{tabular}

Note: b: C. burmannii; c: C. cassia; d: C. camphora; g: C. glanduliferum; i: C. inunctum; k: C. kotoense; o: C. osmophloeum; p: C. parthenoxylon; q: C. philippinense; r: C. reticulatum; s: C. subavenium; t: C. tenuifolium; w: C. wilsonii; z: C. zeylanicum. The same is given in Figure 1.

Some monoterpenes were reported to show significant anti-inflammatory effects. Cyclooxygenase-2 (COX-2) is a ratelimiting enzyme in prostaglandin biosynthesis, which plays a key role in inflammation. In human macrophage-like U937 cells, compound $\mathbf{4}$ was shown to suppress the LPS-induced expression of COX-2 and activate the peroxisome proliferator- 

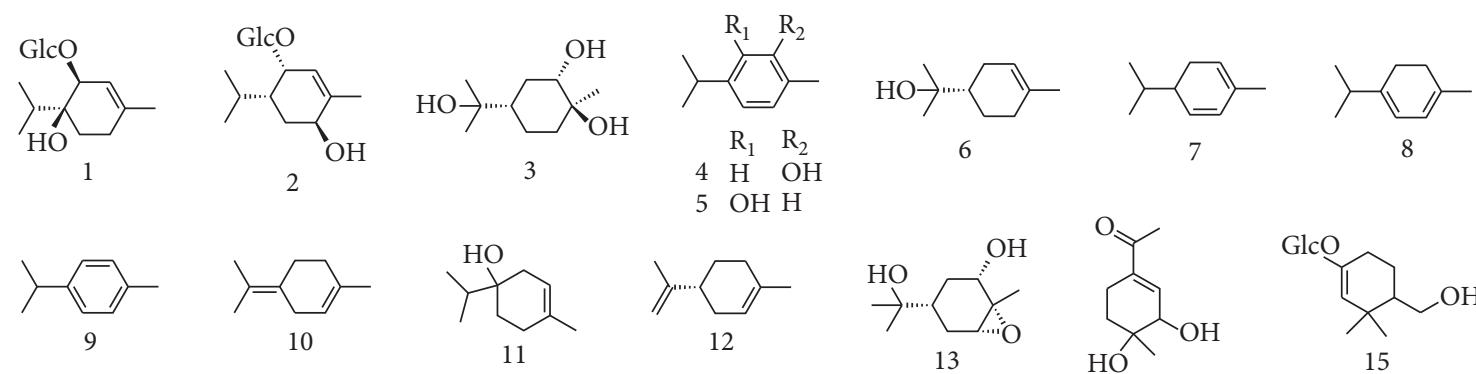<smiles>CC(=O)C1=CC(O)C(O)(O)CC1</smiles><smiles>CC1(C)C=C(Cl)CCC1CO</smiles>

15

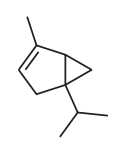

16
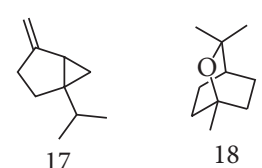

18

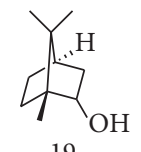

19
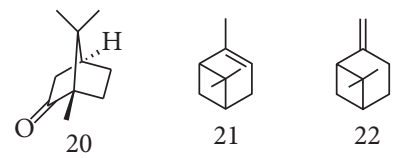

22

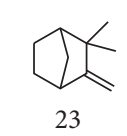

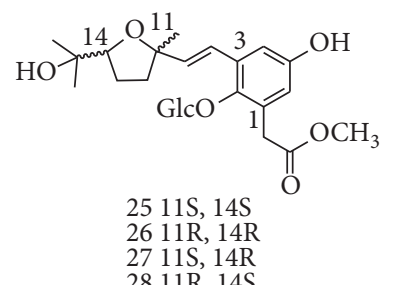<smiles>COC(=O)Cc1cc(O)cc(C/C=C(\C)CC[C@H](O)C(C)(C)O)c1Cl</smiles><smiles>COC(=O)Cc1cc(O)cc(CC2(C)CC(C)(O)CO2)c1CC(=O)O</smiles><smiles>CC(O)C(O)CC(O)C1(C)OC(=O)C1(C)O</smiles>
$2811 \mathrm{R}, 14 \mathrm{~S}$

29
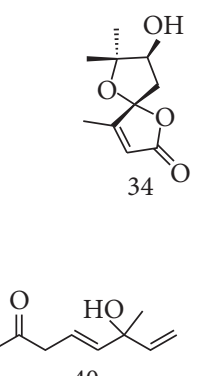

40

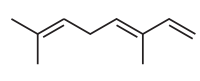

41<smiles>C=CC(=C)CCC=C(C)C</smiles>

42
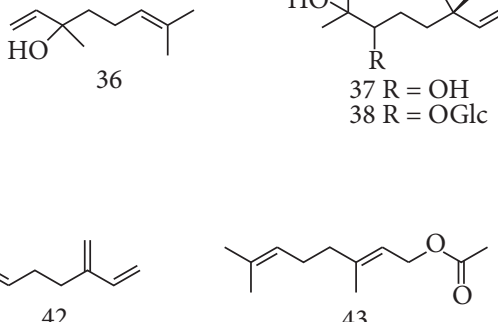

Figure 1: The structures of monoterpenes from Cinnamomum.

activated receptors (PPAR) $\alpha$ and $\gamma$ [34]. Furthermore, 4 could also inhibit the production and actions of NO [25]. Moreover, $\mathrm{NF}-\kappa \mathrm{B}$ has been reported to play an important role in the inflammatory response and LPS/GalN-induced liver injury [35]. The effects of linalool (29) on NF- $\kappa \mathrm{B}$ expression were assessed, and it was found that $\mathbf{2 9}$ could significantly inhibit LPS-induced p65 NF- $\kappa \mathrm{B}$ translocation into the nucleus and $\mathrm{I} \kappa \mathrm{B} \alpha$ degradation. In addition to the NF- $\kappa \mathrm{B}$ inhibition, compound 29 could also induce antioxidant defense via Nrf2 activating and consequently protect against LPS/GalN-induced liver injury [36]. Other than this, another study demonstrated that 29 protected against CS-induced lung inflammation through inhibiting CS-induced NF- $\kappa$ B activation [37].

The anticancer activity of linalool (29) against human prostate cancer (DU145) cells was evaluated by the MTT assay and it was observed that $4.36 \%, 11.54 \%, 21.88 \%$, and $15.54 \%$ of the cells underwent early apoptosis after treatment with $0,20,40$, and $80 \mu \mathrm{M}$ of 29 , respectively [38]. In addition, Okumura et al. confirmed that terpinolene (10) could markedly reduce the expression of protein kinase AKT, which can mediate cell proliferation and survival signals, and contribute to cancer progression [39].
In an immunosuppression assay, Zeng et al. found that monoterpenes 18, 19, and 23 exhibited potent immunosuppressive activities against murine lymphocytes. Compound $\mathbf{2 3}$ inhibited the proliferation of T cells and B cells with an inhibitory ratio of $36.1 \%$ and $20.3 \%$, respectively, at a concentration of $400 \mu \mathrm{M}$ [17]. In another assay, the effect of $\alpha$-terpineol (6) on the generation of nitric oxide in macrophages induced by LPS was evaluated. The nitrite production induced by LPS in these cells was reduced at all concentrations of compound 4 tested $(1,10$, and $100 \mu \mathrm{g} /$ $\mathrm{mL}$ ) [40]. Rufino et al. reported that $\alpha$-pinene (16) could decrease NO production in a dose-dependent manner in primary human chondrocytes, showing the highest inhibition of NO production at a dose of $200 \mu \mathrm{g} / \mathrm{mL}$, with a decrease to $31.5 \%$ relative to cells treated with IL- $1 \beta$ alone [41].

2.2. Sesquiterpenes. A total of 83 sesquiterpenoids (44-126) were isolated from Cinnamomum plants, which contain multiple structural types (Table 2 and Figure 2).

There are 28 ionone sesquiterpenoids (44-71) among these constituents, which are the most abundant 
TABle 2: Sesquiterpenes from the genus Cinnamomum.

\begin{tabular}{|c|c|c|c|c|c|c|}
\hline No. & Type & Compound & $\begin{array}{l}\text { Molecular } \\
\text { formula }\end{array}$ & $\begin{array}{c}\text { Molecular } \\
\text { weight }\end{array}$ & Origin & References \\
\hline 44 & \multirow{28}{*}{ Inone } & Wilsonol A & $\mathrm{C}_{13} \mathrm{H}_{24} \mathrm{O}_{4}$ & 244 & $\mathrm{w}$ & {$[42]$} \\
\hline 45 & & Wilsonol B & $\mathrm{C}_{13} \mathrm{H}_{24} \mathrm{O}_{4}$ & 244 & $\mathrm{w}$ & {$[42]$} \\
\hline 46 & & Wilsonol C & $\mathrm{C}_{13} \mathrm{H}_{22} \mathrm{O}_{4}$ & 242 & $\mathrm{w}$ & {$[42]$} \\
\hline 47 & & Wilsonol D & $\mathrm{C}_{13} \mathrm{H}_{24} \mathrm{O}_{3}$ & 228 & $\mathrm{w}$ & {$[42]$} \\
\hline 48 & & $(3 \mathrm{~S}, 4 \mathrm{~S}, 5 \mathrm{~S}, 6 \mathrm{~S}, 9 \mathrm{~S})-\mathrm{Wilsonol} \mathrm{E}$ & $\mathrm{C}_{13} \mathrm{H}_{26} \mathrm{O}_{3}$ & 230 & $\mathrm{w}$ & {$[42]$} \\
\hline 49 & & (3S,4S,5S,6S,9R)-Wilsonol E & $\mathrm{C}_{13} \mathrm{H}_{26} \mathrm{O}_{3}$ & 230 & $\mathrm{w}$ & {$[42]$} \\
\hline 50 & & Wilsonol F & $\mathrm{C}_{13} \mathrm{H}_{26} \mathrm{O}_{3}$ & 230 & $\mathrm{w}$ & [42] \\
\hline 51 & & Wilsonol G & $\mathrm{C}_{13} \mathrm{H}_{24} \mathrm{O}_{4}$ & 244 & s, w & {$[8,42]$} \\
\hline 52 & & $\begin{array}{c}(3 \mathrm{~S}, 4 \mathrm{~S}, 5 \mathrm{~S}, 6 \mathrm{~S}, 9 \mathrm{~S})-3,4 \text {-Dihydroxy-5, } \\
\text { 6-dihydro- } \beta \text {-ionol }\end{array}$ & $\mathrm{C}_{13} \mathrm{H}_{24} \mathrm{O}_{3}$ & 228 & $\mathrm{w}$ & {$[42]$} \\
\hline 53 & & Lasianthionoside A & $\mathrm{C}_{19} \mathrm{H}_{32} \mathrm{O}_{9}$ & 404 & $\mathrm{w}$ & {$[42]$} \\
\hline 54 & & Boscialin & $\mathrm{C}_{13} \mathrm{H}_{22} \mathrm{O}_{3}$ & 226 & $\mathrm{c}$ & {$[43]$} \\
\hline 55 & & $\begin{array}{c}\text { (3S,5R,6S,7E)-Megasfigma-7-ene-3, } \\
\text { 5,6,9-tetrol }\end{array}$ & $\mathrm{C}_{13} \mathrm{H}_{24} \mathrm{O}_{4}$ & 244 & $\mathrm{~s}, \mathrm{w}$ & {$[8,42]$} \\
\hline 56 & & $\begin{array}{c}\text { (3S,5S,6S,9R)-3,6-Dihydroxy-5, } \\
\text { 6-dihydro- } \beta \text {-ionol }\end{array}$ & $\mathrm{C}_{13} \mathrm{H}_{24} \mathrm{O}_{3}$ & 228 & $\mathrm{w}$ & {$[42]$} \\
\hline 57 & & Wilsonol $\mathrm{H}$ & $\mathrm{C}_{13} \mathrm{H}_{24} \mathrm{O}_{5}$ & 260 & s, w & {$[8]$} \\
\hline 58 & & Wilsonol I & $\mathrm{C}_{13} \mathrm{H}_{24} \mathrm{O}_{3}$ & 228 & $\mathrm{w}$ & {$[42]$} \\
\hline 59 & & Wilsonol J & $\mathrm{C}_{13} \mathrm{H}_{24} \mathrm{O}_{3}$ & 228 & $\mathrm{w}$ & {$[42]$} \\
\hline 60 & & Wilsonol K & $\mathrm{C}_{13} \mathrm{H}_{22} \mathrm{O}_{3}$ & 226 & $\mathrm{w}$ & {$[42]$} \\
\hline 61 & & Wilsonol L & $\mathrm{C}_{13} \mathrm{H}_{22} \mathrm{O}_{3}$ & 226 & $\mathrm{w}$ & {$[42]$} \\
\hline 62 & & $\begin{array}{l}\text { (3R,9S)-Megastigman-5-ene-3, } \\
\text { 9-diol 3-O- } \beta \text {-D-glucopyranoside }\end{array}$ & $\mathrm{C}_{19} \mathrm{H}_{34} \mathrm{O}_{7}$ & 374 & $\mathrm{w}$ & {$[42]$} \\
\hline 63 & & $\begin{array}{c}\text { (3S,4R,9R)-3,4,9-Trihydroxymegastigman } \\
-5 \text {-ene }\end{array}$ & $\mathrm{C}_{13} \mathrm{H}_{24} \mathrm{O}_{3}$ & 228 & $\mathrm{w}$ & {$[42]$} \\
\hline 64 & & Apocynol A & $\mathrm{C}_{13} \mathrm{H}_{20} \mathrm{O}_{3}$ & 224 & $\mathrm{w}$ & [42] \\
\hline 65 & & Blumenol A & $\mathrm{C}_{13} \mathrm{H}_{20} \mathrm{O}_{3}$ & 224 & $c, p$ & {$[43,44]$} \\
\hline 66 & & Dehydrovomifoliol & $\mathrm{C}_{13} \mathrm{H}_{18} \mathrm{O}_{3}$ & 222 & c & [43] \\
\hline 67 & & $\begin{array}{c}\text { (3S,5R,6S,9S)-3,6,9-Trihydroxymegastigman } \\
\text {-7-ene 3-O- } \beta \text {-D-glucopyranoside }\end{array}$ & $\mathrm{C}_{19} \mathrm{H}_{34} \mathrm{O}_{8}$ & 390 & c & {$[19]$} \\
\hline 68 & & $\begin{array}{l}\text { (1R,2R)-4-[(3S)-3-Hydroxybutyl]-3,3,5- } \\
\text { trimethylcyclohex-4-ene-1,2-diol }\end{array}$ & $\mathrm{C}_{13} \mathrm{H}_{24} \mathrm{O}_{3}$ & 228 & c & {$[45]$} \\
\hline 69 & & $\begin{array}{c}\text { (3S,5R,6S,7E)-3,5,6-Trihydroxy-7 } \\
\text {-megastigmen-9-one }\end{array}$ & $\mathrm{C}_{13} \mathrm{H}_{22} \mathrm{O}_{4}$ & 242 & c & {$[46]$} \\
\hline 70 & & Grasshopper ketone & $\mathrm{C}_{13} \mathrm{H}_{20} \mathrm{O}_{3}$ & 224 & c & {$[43]$} \\
\hline 71 & & Asicariside B1 & $\mathrm{C}_{18} \mathrm{H}_{30} \mathrm{O}_{8}$ & 374 & $\mathrm{~s}$ & {$[8]$} \\
\hline $\begin{array}{l}72 \\
73 \\
\end{array}$ & Caryophyllane & $\begin{array}{l}\text { Caryophyllene oxide } \\
\text { trans-Caryophyllene }\end{array}$ & $\begin{array}{c}\mathrm{C}_{15} \mathrm{H}_{24} \mathrm{O} \\
\mathrm{C}_{15} \mathrm{H}_{24} \\
\end{array}$ & $\begin{array}{l}220 \\
204\end{array}$ & $\begin{array}{c}\mathrm{O}, \mathrm{s} \\
\mathrm{c}\end{array}$ & $\begin{array}{c}{[9,16]} \\
{[47]} \\
\end{array}$ \\
\hline 74 & \multirow[t]{2}{*}{ Humulane } & $\begin{array}{l}\text { (2E,9E)-6,7-cis-Dihydroxyhumulan } \\
\text {-2,9-diene }\end{array}$ & $\mathrm{C}_{15} \mathrm{H}_{26} \mathrm{O}_{2}$ & 238 & c & {$[46]$} \\
\hline 75 & & $\alpha$-Humulene & $\mathrm{C}_{15} \mathrm{H}_{24}$ & 204 & g & {$[10]$} \\
\hline 76 & \multirow{13}{*}{ Cadinane } & Cinnamoid B & $\mathrm{C}_{15} \mathrm{H}_{24} \mathrm{O}_{3}$ & 252 & c & {$[48,49]$} \\
\hline 77 & & Cinnamoid C & $\mathrm{C}_{15} \mathrm{H}_{24} \mathrm{O}_{3}$ & 252 & c & {$[48,49]$} \\
\hline 78 & & Mustakone & $\mathrm{C}_{15} \mathrm{H}_{24} \mathrm{O}_{3}$ & 252 & c & {$[46]$} \\
\hline 79 & & Oxyphyllenodiol A & $\mathrm{C}_{14} \mathrm{H}_{22} \mathrm{O}_{3}$ & 238 & s & {$[50]$} \\
\hline 80 & & Oxyphyllenodiol B & $\mathrm{C}_{14} \mathrm{H}_{22} \mathrm{O}_{3}$ & 238 & s & {$[50]$} \\
\hline 81 & & (-)-15-Hydroxytmuurolol & $\mathrm{C}_{15} \mathrm{H}_{26} \mathrm{O}_{2}$ & 238 & c & [49] \\
\hline 82 & & 15 -Hydroxy- $\alpha$-cadinol & $\mathrm{C}_{15} \mathrm{H}_{26} \mathrm{O}_{2}$ & 238 & c & {$[49]$} \\
\hline 83 & & $\beta$-Cadinene & $\mathrm{C}_{15} \mathrm{H}_{24}$ & 204 & $\mathrm{z}$ & [11] \\
\hline 84 & & $\delta$-Cadinene & $\mathrm{C}_{15} \mathrm{H}_{24}$ & 204 & $\mathrm{c}, \mathrm{d}, \mathrm{o}, \mathrm{z}$ & [10-13] \\
\hline 85 & & $\alpha$-Muurolene & $\mathrm{C}_{15} \mathrm{H}_{24}$ & 204 & $c, d$ & [14] \\
\hline 86 & & $\alpha$-Cadinol & $\mathrm{C}_{15} \mathrm{H}_{26} \mathrm{O}$ & 222 & $c, d$ & [14] \\
\hline 87 & & $\alpha$-Calacorene & $\mathrm{C}_{15} \mathrm{H}_{20}$ & 200 & $c, o, z$ & {$[11,12]$} \\
\hline 88 & & Calamenene & $\mathrm{C}_{15} \mathrm{H}_{22}$ & 202 & $\mathrm{z}$ & [11] \\
\hline
\end{tabular}


TABle 2: Continued.

\begin{tabular}{|c|c|c|c|c|c|c|}
\hline No. & Type & Compound & $\begin{array}{l}\text { Molecular } \\
\text { formula }\end{array}$ & $\begin{array}{c}\text { Molecular } \\
\text { weight }\end{array}$ & Origin & References \\
\hline 89 & \multirow{8}{*}{ Eudesmane } & 4(15)-Eudesmene-1 $\beta, 7,11$-triol & $\mathrm{C}_{15} \mathrm{H}_{26} \mathrm{O}_{3}$ & 254 & c & {$[51]$} \\
\hline 90 & & $1 \beta, 6 \alpha$-Dihydroxyeudesm-4(15)-ene & $\mathrm{C}_{15} \mathrm{H}_{26} \mathrm{O}_{2}$ & 238 & c & {$[51]$} \\
\hline 91 & & $\begin{array}{l}1 \alpha, 6 \beta \text {-Dihydroxy-5,10-bis-epi-eudesm-15- } \\
\text { carboxaldehyde- } 6 \text {-O- } \beta \text {-D-glucopyranoside }\end{array}$ & $\mathrm{C}_{21} \mathrm{H}_{36} \mathrm{O}_{9}$ & 433 & s & {$[8]$} \\
\hline 92 & & $1 \beta, 4 \beta, 11$-Trihydroxyl-6 $6 \beta$-gorgonane & $\mathrm{C}_{15} \mathrm{H}_{28} \mathrm{O}_{3}$ & 256 & c & {$[51]$} \\
\hline 93 & & Cinnamosim B & $\mathrm{C}_{15} \mathrm{H}_{24} \mathrm{O}_{4}$ & 270 & c & {$[51]$} \\
\hline 94 & & Cinnamosim A & $\mathrm{C}_{15} \mathrm{H}_{24} \mathrm{O}_{3}$ & 252 & c & {$[51]$} \\
\hline 95 & & 1 $\beta, 7$-Dihydroxyl opposit-4(15)-ene & $\mathrm{C}_{15} \mathrm{H}_{26} \mathrm{O}_{2}$ & 238 & c & {$[51]$} \\
\hline 96 & & $1 \beta, 11$-Dihydroxyl opposit-4(15)-ene & $\mathrm{C}_{15} \mathrm{H}_{26} \mathrm{O}_{2}$ & 238 & c & {$[51]$} \\
\hline 97 & \multirow{4}{*}{ Guaiane } & $4 \alpha-10 \alpha$-Dihydroxy-5 $\beta$-H-guaja-6-ene & $\mathrm{C}_{15} \mathrm{H}_{26} \mathrm{O}_{2}$ & 238 & c & {$[51]$} \\
\hline 98 & & Alpinenone & $\mathrm{C}_{15} \mathrm{H}_{26} \mathrm{O}_{2}$ & 238 & $\mathrm{t}$ & {$[52]$} \\
\hline 99 & & $\alpha$-Bulnesene & $\mathrm{C}_{15} \mathrm{H}_{24}$ & 204 & c & {$[53]$} \\
\hline 100 & & Guaiol & $\mathrm{C}_{15} \mathrm{H}_{26} \mathrm{O}$ & 222 & $\mathrm{~g}$ & {$[10]$} \\
\hline 101 & \multirow{6}{*}{ Aromadendrane } & Aromadendrane- $4 \beta, 10 \alpha$-diol & $\mathrm{C}_{15} \mathrm{H}_{26} \mathrm{O}_{2}$ & 238 & c & {$[51]$} \\
\hline 102 & & Aromadendrane- $4 \alpha, 10 \alpha$-diol & $\mathrm{C}_{15} \mathrm{H}_{26} \mathrm{O}_{2}$ & 238 & c & {$[51]$} \\
\hline 103 & & 1-Epimeraromadendrane- $4 \beta, 10 \alpha$-Diol & $\mathrm{C}_{15} \mathrm{H}_{26} \mathrm{O}_{2}$ & 238 & c & {$[51]$} \\
\hline 104 & & Espatulenol & $\mathrm{C}_{15} \mathrm{H}_{24} \mathrm{O}$ & 220 & c & {$[14]$} \\
\hline 105 & & Spathulenol & $\mathrm{C}_{15} \mathrm{H}_{24} \mathrm{O}$ & 220 & o & [12] \\
\hline 106 & & $(-)$-Isoledene & $\mathrm{C}_{15} \mathrm{H}_{24}$ & 204 & $\mathrm{c}$ & {$[53]$} \\
\hline 107 & \multirow{3}{*}{ Bisabolane } & $\beta$-Bisabolene & $\mathrm{C}_{15} \mathrm{H}_{24}$ & 204 & c & {$[14]$} \\
\hline 108 & & $\alpha$-Bisabolol & $\mathrm{C}_{15} \mathrm{H}_{26} \mathrm{O}_{2}$ & 238 & $c$ & {$[14]$} \\
\hline 109 & & Curcumene & $\mathrm{C}_{15} \mathrm{H}_{22}$ & 202 & $\mathrm{c}, \mathrm{o}$ & {$[14]$} \\
\hline 110 & Germacrane & Germacrene D & $\mathrm{C}_{15} \mathrm{H}_{28}$ & 208 & $\mathrm{c}$ & {$[47]$} \\
\hline 111 & Cedrane & Cedrene & $\mathrm{C}_{15} \mathrm{H}_{24}$ & 204 & $\mathrm{c}$ & {$[20]$} \\
\hline 112 & \multirow{15}{*}{ Others } & 3S-(+)-9-Oxonerolidol & $\mathrm{C}_{16} \mathrm{H}_{26} \mathrm{O}$ & 234 & $\mathrm{~d}$ & {$[21]$} \\
\hline 113 & & 2,6,11-Trimethyldodeca-2,6,10-triene & $\mathrm{C}_{15} \mathrm{H}_{26}$ & 206 & $\mathrm{q}$ & {$[54]$} \\
\hline 114 & & $(+)-(6 \mathrm{~S}, 7 \mathrm{E}, 9 \mathrm{Z})$-Abscisic ester & $\mathrm{C}_{16} \mathrm{H}_{22} \mathrm{O}_{4}$ & 278 & $\mathrm{w}$ & {$[42]$} \\
\hline 115 & & Gibberodione & $\mathrm{C}_{15} \mathrm{H}_{24} \mathrm{O}_{2}$ & 236 & s & [55] \\
\hline 116 & & $(+)$-Abscisic acid & $\mathrm{C}_{15} \mathrm{H}_{20} \mathrm{O}_{4}$ & 264 & $b, s$ & {$[56,57]$} \\
\hline 117 & & Caryolane-1,9 $\beta$-diol & $\mathrm{C}_{15} \mathrm{H}_{26} \mathrm{O}_{2}$ & 238 & $c$ & {$[46,51]$} \\
\hline 118 & & Vlovane- $2 \beta, 9 \alpha$-diol & $\mathrm{C}_{15} \mathrm{H}_{26} \mathrm{O}_{2}$ & 238 & c & [46] \\
\hline 119 & & Cinnamoid A & $\mathrm{C}_{15} \mathrm{H}_{26} \mathrm{O}_{2}$ & 238 & c & {$[48,49]$} \\
\hline 120 & & Cinnamoid D & $\mathrm{C}_{15} \mathrm{H}_{24} \mathrm{O}_{2}$ & 236 & c & {$[48,49]$} \\
\hline 121 & & Cinnamoid E & $\mathrm{C}_{15} \mathrm{H}_{22} \mathrm{O}_{2}$ & 234 & c & {$[48,49]$} \\
\hline 122 & & (-)-15-Hydroxy-T-muurolol & $\mathrm{C}_{15} \mathrm{H}_{22} \mathrm{O}_{2}$ & 218 & c & {$[13]$} \\
\hline 123 & & $\alpha$-Cubebene & $\mathrm{C}_{15} \mathrm{H}_{24}$ & 204 & c & [53] \\
\hline 124 & & $\alpha$-Copaene & $\mathrm{C}_{15} \mathrm{H}_{24}$ & 204 & $c, o, z$ & {$[11,12]$} \\
\hline 125 & & 1-(3-Indolyl)-2,3-dihydroxypropan-1-one & $\mathrm{C}_{15} \mathrm{H}_{26} \mathrm{O}_{2}$ & 238 & c & [43] \\
\hline 126 & & Patchouli alcohol & $\mathrm{C}_{15} \mathrm{H}_{26} \mathrm{O}$ & 222 & $\mathrm{c}$ & [53] \\
\hline
\end{tabular}

sesquiterpene skeletons from the genus Cinnamomum. Although ionone sesquiterpenes are common in natural products, this class of compounds from Cinnamomum has its special features, which are demonstrated as follows: (1) C-4 is connected to a hydroxyl group in some compounds, including 44-46, 48, 49, 51-53, 57, and 63. (2) The methyl group attached to C-5 is hydroxylated, such as compounds 47, 58-61, and 64. (3) The hydroxyl group of C-3 was removed in compound 51. (4) C-2 was connected to a hydroxyl group, such as compounds $\mathbf{5 1}$ and $\mathbf{6 8}$.

Compounds 72 and 73 belong to caryophyllane sesquiterpenes. Compounds $\mathbf{7 4}$ and $\mathbf{7 5}$ belong to humulane sesquiterpenes with an eleven-membered ring. Compounds 97-100 are guaiane sesquiterpenes, among which 98 was further cyclized on the basis of this structure and has exerted potent antitumor activity.
Cadinane sesquiterpenes include compounds 76-88. The methyl groups attached to the C-9 positions of $\mathbf{7 9}$ and $\mathbf{8 0}$ were degraded, and both of them are derived from $C$. subavenium. In compounds $\mathbf{8 1}$ and $\mathbf{8 2}$, which were isolated from C. cassia, the hydrogen at the C-10 position was hydroxylated. In addition, compounds $\mathbf{8 3 - 8 8}$ are common in the volatile oils of Cinnamomum plants.

Eudesmane sesquiterpenes include compounds 89-96. Compound 92 is a rearranged sesquiterpene, with its isopropanol group migrating from C-7 to C-6. Furthermore, 94-96 are also rearranged sesquiterpenes, with one carbon migrating to form a cyclopentane instead of cyclohexane. Interestingly, the compounds above were all derived from $C$. cassia.

Compounds 101-106 are aromadendrane sesquiterpenes. Furthermore, compounds 107-111 are the common components of the volatile oils in Cinnamomum plants. 


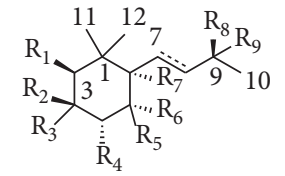

$\begin{array}{llllll}\mathrm{R}_{1} & \mathrm{R}_{2} & \mathrm{R}_{3} & \mathrm{R}_{4} & \mathrm{R}_{5} & \mathrm{R}_{6}\end{array}$

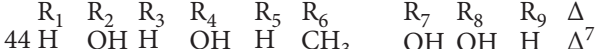
$\begin{array}{llllllll}45 \mathrm{H} & \mathrm{OH} \mathrm{H} & \mathrm{OH} & \mathrm{H} & \mathrm{CH}_{3} & \mathrm{OH} \mathrm{H} & \mathrm{OH} \Delta_{7}^{7}\end{array}$ $46 \mathrm{H} \quad \mathrm{OH} \mathrm{H} \quad \mathrm{OH} \quad \mathrm{H} \quad \mathrm{CH}_{3} \quad \mathrm{OH}=\mathrm{O} \Delta^{7}$

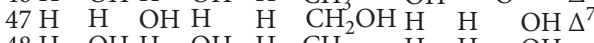
$\begin{array}{lllllllll}48 \mathrm{H} & \mathrm{OH} \mathrm{H} & \mathrm{OH} & \mathrm{H} & \mathrm{CH}_{3} & \mathrm{H} & \mathrm{H} & \mathrm{OH}\end{array}$ $\begin{array}{llllllll}49 \mathrm{H} & \mathrm{OH} \mathrm{H} & \mathrm{OH} & \mathrm{H} & \mathrm{CH}_{3} & \mathrm{H} & \mathrm{OH} & \mathrm{H}\end{array}$

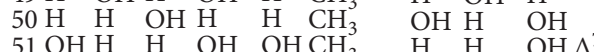

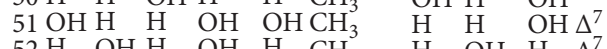

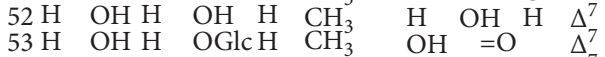

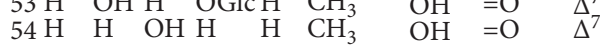<smiles>CC(=O)C=CC1(C)C(C)(C)CC(O)CC1(C)O</smiles><smiles>CC(=O)/C=C\C1C(C)(C)CC(O)CC1(C)O</smiles><smiles>CC(=O)C=C1C(C)(C)CC(Cl)CC1(C)O</smiles>

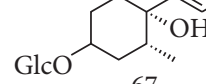
67<smiles>[R]C1CC(C)(C)C([R])(C)C([R3])(C)C1(C)C=CC(C)O</smiles>

$\begin{array}{llll}\mathrm{R}_{1} & \mathrm{R}_{2} & \mathrm{R}_{3} & \mathrm{R}_{4}\end{array}$ $55 \mathrm{OH} \mathrm{H}^{\mathrm{H}} \mathrm{OH}$ $56 \mathrm{H} \quad \mathrm{OH} \mathrm{H} \quad \mathrm{H}$ $57 \mathrm{OH} \mathrm{H} \quad \mathrm{OH} \mathrm{OH}$<smiles>CC(O)/C=C/CC(C)(C)C</smiles><smiles>[R3]C1=C(/C=C/C([R])([R3])C)C(C)(C)CC([R])C1[R]</smiles>

$\mathrm{R}_{1} \mathrm{R}_{2}$ $59 \mathrm{OH} \quad \mathrm{H} \quad \mathrm{CH}_{2} \mathrm{OH} \quad \mathrm{H} \quad \mathrm{OH}$ $60 \mathrm{OH} \quad \mathrm{H} \quad \mathrm{CH}_{2} \mathrm{OH} \quad \mathrm{OH} \mathrm{H} \quad \Delta^{7}$ $61 \mathrm{OH} \quad \mathrm{H} \quad \mathrm{CH}_{2} \mathrm{OH} \quad \mathrm{H} \quad \mathrm{OH} \Delta^{7}$ 62 OGlc $\mathrm{H} \quad \mathrm{CH}_{3} \quad \mathrm{OH} \mathrm{H}$ $63 \mathrm{OH} \quad \mathrm{OH} \mathrm{CH}_{3}$ $\mathrm{H} \mathrm{OH}$<smiles>CC(=O)CCC1C(=O)C=C(C(C)C)CCC1C</smiles><smiles>[R2]CC1=CC(=O)CC(C)(C)C1/C=C/C([R1])([R3])C</smiles>

$\begin{array}{lllll}\mathrm{R}_{1} & \mathrm{R}_{2} & \mathrm{R}_{3} & \mathrm{R}_{4}\end{array}$ $64 \mathrm{H} \quad \mathrm{OH} \mathrm{OH} \mathrm{H}$ $65 \mathrm{OH} \mathrm{H} \mathrm{OH} \mathrm{H}$ $66 \mathrm{OH} \mathrm{H}=\mathrm{O}$<smiles>CC1=C(CCC(C)O)C(C)(C)C(O)[C@H](O)C1</smiles><smiles>C=C1CCC2O[C@H]2CCC(C)(C)C1CC</smiles><smiles>CC1CCC(C)C(C(C)C)CC1</smiles><smiles>[2H]C1([2H])CC(O)C(C)(O)CCC1CC(C)C</smiles><smiles>CC1(C)CC(O)C2CC[C@H](O)C[C@H]2C1</smiles><smiles>CC(C)=CCCC(O)C(C)(O)CC=CC(C)(C)O</smiles><smiles>CCC(=O)O</smiles>

74<smiles>CC1=CCC(C)(C)CCCC(C)=CCC1</smiles>

80

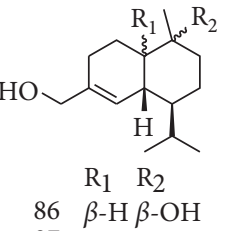
$87 \alpha-\mathrm{H} \alpha-\mathrm{OH}$<smiles>CC1=CCC2C(C)=CCC(C(C)C)C2C1</smiles>

88<smiles>CC1=CC2(C(C)C)CCCC2=C(C)CC1</smiles>

$81 \mathrm{R}=\alpha-\mathrm{OH}$ $82 \mathrm{R}=\beta-\mathrm{OH}$

83<smiles>CC1=CC2(C(C)C)C(CCC1C)CCC2(C)O</smiles><smiles>CC1=CCC(C(C)C)c2cc(C)ccc21</smiles><smiles>Cc1ccc2c(c1)C(C(C)C)CCC2C</smiles>

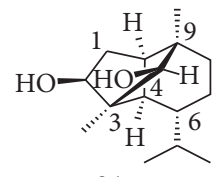

94<smiles>C=C1C(O)C2(O)C3CC(O)C(CC3C(C)C)C12</smiles><smiles>CC1CC2CC(C(C)(C)O)CC(C1=O)C2C</smiles><smiles>CC1=CC(=O)C2CC1C(C(C)C)C2</smiles><smiles>[R19]C1CCC2(CCC(O)CCC2=C)C1([R3])C</smiles>
$\begin{array}{llll}R_{1} & R_{2} & R_{3}\end{array}$ $98 \mathrm{OH} \mathrm{OH} \mathrm{H}$<smiles>CC(O)[C@H]1CCC[C@]2(C)C(O)CC[C@](C)(O)[C@H]1[C@@H]2OO</smiles><smiles>CC12CC[C@H](O)[C@H](O)C1=CC(O)(O)CC2</smiles>

01

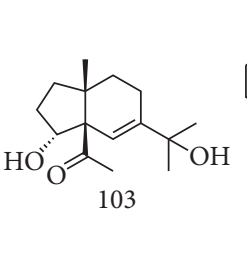<smiles>[R]C([R]([R])([H])[H])C([R])(C)C1CC[C@]2(C)C(O)CCC(=C)[C@H]12</smiles><smiles>CC1=CC[C@]23CC[C@@H](CCC2C(C)C)[C@@]13C</smiles><smiles>CC1=CCC2C(Br)C2CCC1C(C)C</smiles><smiles>CC(C)C1=CC2C(C)CCC2[C@H](O)CC1</smiles>
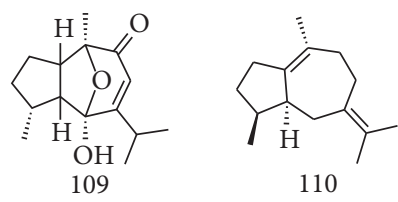

110
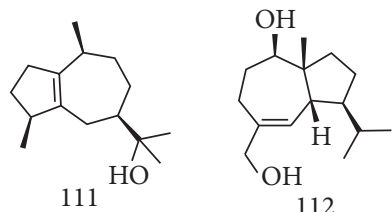

(a)

Figure 2: Continued. 


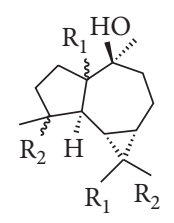

$113 \beta-\mathrm{H} \alpha-\mathrm{OH}$

$114 \alpha-\mathrm{H} \alpha-\mathrm{OH}$

$115 \beta-\mathrm{H} \beta-\mathrm{OH}$

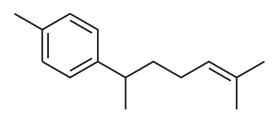

121

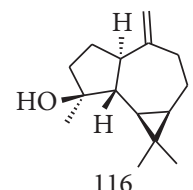

116

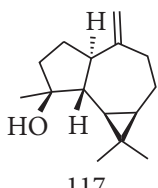

117

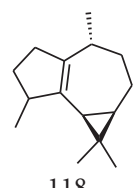

118

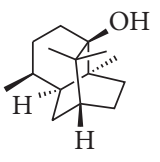

123

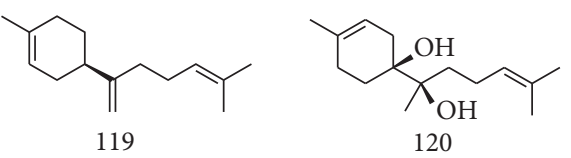

120
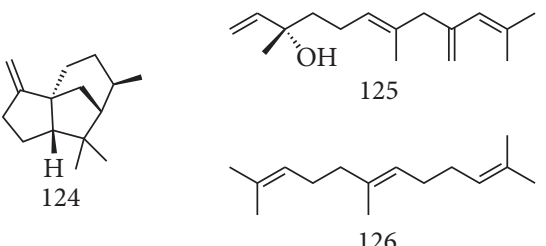

(b)

Figure 2: The structures of sesquiterpenes from Cinnamomum.

Among these, 107-109 are bisabolane sesquiterpenes, while 110 and 111 belong to germacrane and cedrane sesquiterpenes, respectively. Compound 117 is the result of the further cyclization based on the caryophyllane skeleton, and 118 was formed through the migration of one carbon atom based on the structure of 117 .

In 2014, Yan et al. separated cinnamonoid A (119) from C. cassia with an unprecedented skeleton, which may be derived from cadinane sesquiterpenes. The possible biosynthesis pathway of $\mathbf{1 1 9}$ was carried out by the cleavage of C-5 and C-6, followed by the construction of C-4 with C- 6 and C-5 with C-10. Furthermore, compounds 120-122 are rearranged cadinane sesquiterpenes with the cleavage of C-9 and C-4.

Some sesquiterpenoids were reported to show significant anti-inflammatory effects. Tung et al. confirmed that the essential oil of C. osmophloeum twig exhibited a potent inhibition on NO production in LPS-stimulated RAW 264.7 macrophage cells. The anti-inflammatory effects of some constituents from essential oils were also evaluated. Compound $72(54.0 \%)$ exhibited stronger inhibition activities than twig essential oil (48.3\%) at the concentration of $10 \mu \mathrm{g} /$ $\mathrm{mL}$, while 117 exhibited an activity similar to that of twig essential oil, with inhibition rates of 46.1 and $50.9 \%$, respectively [12]. In addition, Guoruoluo et al. evaluated some sesquiterpenoids for the antimicrobial activities. Compounds including 89, 90, 93-95, 97, 101, 102, and 117 showed significant antimicrobial activities against Candida albicans with inhibitory zone diameters ranging from 8 to $11 \mathrm{~mm}$. Moreover, 90, 101, and 117 have exhibited moderate inhibitory effects on Escherichia coli and Staphylococcus aureus with inhibitory zone diameters ranging from 7 to $11 \mathrm{~mm}$ [51]. In another antibacterial assay, the activities of $\mathbf{7 9}$ and $\mathbf{8 0}$ against Candida glabrata were also evaluated, and the MIC values were 187.6 and 177.5 , respectively [58].

2.3. Diterpenes. A total of 55 diterpenes were isolated from Cinnamomum plants (Table 3 and Figure 3). Most of these were derived from C. cassia (127-178), and $\mathbf{1 7 9}$ was derived from $C$. kotoense and C. philippinense. Nine new diterpene skeletons were found from this species, including ryanodane (cinncassiol B type), 11,12-seco-ryanodane (cinncassiol A type), 7,8-secoryanodane (cinncassiol $\mathrm{C}$ type), isoryanodane (cinncassiol D type), 10,13-cyclo-12,13-seco-isoryanodane (cinncassiol E type), 12,13-seco-isoryanodane (cinncassiol $\mathrm{F}$ type), 11,12seco-isoryanodane (cinncassiol G type), 6,10-cyclo-12,13-secoisoryanodane (cinnamomane), and 11,14-cyclo-8,14:12,13-diseco-isoryanodane (cassiabudane).

Among these nine skeletons, the representative compounds of the cinncassiol A-G types were 127, 136, 141, 143-145, 148, 150, and 164-166. Furthermore, the typical constituents of cinnamomane-type diterpenes were $\mathbf{1 7 3}$ and 174, while those of cassiabudane-type diterpenes were $\mathbf{1 7 5}$ and 176.

The biogenetic relationships of the nine diterpene skeletons were proposed by Zhou et al. (Figure 4) [69]. Cinncassiol B type (134-140), that is, renolane-type diterpenes, is a 6/5/5/6/5 pentacyclic skeleton with a ketal structure. The two oxygen atoms connected to C-11 were, respectively, connected to hydrogen and C-6. In addition, cinncassiol A-type diterpenes (127-133) have a lactone skeleton produced by the cleavage of the bond between C-11 and C-12, and the formation of the carbon-oxygen double bond at $\mathrm{C}-11$ in cinncassiol B-type diterpenes. The linkage between C-7 and C-8 was cleaved, and two carbonyl groups were formed at the two positions in the cinncassiol B type to produce a diketone skeleton, cinncassiol C type (141-144). Cinncassiol D-type diterpenes (145-163) are isorinolone diterpenes, which have a pentacyclic ring skeleton produced by the migration of the bond between C-5 and C- 6 of cinncassiol B-type diterpenes to the C-1 position. Similarly, cinncassiol G-type diterpenes were formed by the migration of 5,6-bond to C-1 in cinncassiol A-type diterpenes.

The possible biosynthetic pathway of cassiabudane diterpenes was proposed by Zhou et al. [69]. These were formed from 18-hydroxyperseanol (159), an isoryanodane diterpenoid, by the cleavage of the 12,13-bond and migration of the 14,8-bond to C-11 through a series of retro-aldol, aldol, and oxidation reactions. 
tblBody

Table 3: Diterpenes from the genus Cinnamomum.

\begin{tabular}{|c|c|c|c|c|c|}
\hline No. & Type & Compound & Molecular formula & Molecular weight & References \\
\hline 127 & \multirow{7}{*}{ Cinncassiol A } & Cinncassiol A & $\mathrm{C}_{20} \mathrm{H}_{30} \mathrm{O}_{7}$ & 382 & {$[46,49]$} \\
\hline 128 & & Cinncassiol A 19-O- $\beta$-D-glucopyranoside & $\mathrm{C}_{26} \mathrm{H}_{40} \mathrm{O}_{12}$ & 544 & {$[59,60]$} \\
\hline 129 & & Anhydrocinnzeylanine & $\mathrm{C}_{22} \mathrm{H}_{32} \mathrm{O}_{7}$ & 408 & [49] \\
\hline 130 & & Epianhydrocinnzeylanol & $\mathrm{C}_{20} \mathrm{H}_{30} \mathrm{O}_{6}$ & 366 & {$[61]$} \\
\hline 131 & & 1-Acetylcinncassiol A & $\mathrm{C}_{22} \mathrm{H}_{32} \mathrm{O}_{8}$ & 424 & [59] \\
\hline 132 & & 2,3-Dehydroanhydrocinnzeylanine & $\mathrm{C}_{22} \mathrm{H}_{30} \mathrm{O}_{7}$ & 406 & {$[59]$} \\
\hline 133 & & Cinncassiol $\mathrm{H}$ & $\mathrm{C}_{20} \mathrm{H}_{30} \mathrm{O}_{7}$ & 382 & {$[61]$} \\
\hline 134 & \multirow{7}{*}{ Cinncassiol B } & Cinnzeylanol & $\mathrm{C}_{20} \mathrm{H}_{32} \mathrm{O}_{7}$ & 384 & {$[61]$} \\
\hline 135 & & Cinnzeylanine & $\mathrm{C}_{22} \mathrm{H}_{34} \mathrm{O}_{8}$ & 427 & {$[61]$} \\
\hline 136 & & Cinncassiol B & $\mathrm{C}_{20} \mathrm{H}_{32} \mathrm{O}_{8}$ & 400 & {$[46]$} \\
\hline 137 & & Ryanodol & $\mathrm{C}_{20} \mathrm{H}_{32} \mathrm{O}_{8}$ & 400 & {$[46]$} \\
\hline 138 & & Ryanodol 14-monoacetate & $\mathrm{C}_{22} \mathrm{H}_{34} \mathrm{O}_{9}$ & 442 & {$[46]$} \\
\hline 139 & & 18-Hydroxycinnzeylanine & $\mathrm{C}_{22} \mathrm{H}_{34} \mathrm{O}_{9}$ & 442 & [59] \\
\hline 140 & & Cinnzeylanone & $\mathrm{C}_{20} \mathrm{H}_{30} \mathrm{O}_{7}$ & 382 & {$[46]$} \\
\hline 141 & \multirow{4}{*}{ Cinncassiol C } & \multirow{4}{*}{$\begin{array}{c}\text { Cinncassiol } C_{1} \\
\text { Cinncassiol } C_{1}-19-O-\beta-D \\
\text {-glucopyranoside } \\
\text { Cinncassiol } C_{2} \\
\text { Cinncassiol } C_{3}\end{array}$} & $\mathrm{C}_{26} \mathrm{H}_{38} \mathrm{O}_{12}$ & 542 & {$[62]$} \\
\hline 142 & & & $\mathrm{C}_{20} \mathrm{H}_{28} \mathrm{O}_{7}$ & 380 & {$[63]$} \\
\hline 143 & & & $\mathrm{C}_{20} \mathrm{H}_{28} \mathrm{O}_{6}$ & 364 & {$[63]$} \\
\hline 144 & & & $\mathrm{C}_{20} \mathrm{H}_{30} \mathrm{O}_{7}$ & 382 & {$[63]$} \\
\hline 145 & \multirow{19}{*}{ Cinncassiol D } & Cinncassiol $\mathrm{D}_{1}$ & $\mathrm{C}_{26} \mathrm{H}_{42} \mathrm{O}_{10}$ & 515 & {$[64,65]$} \\
\hline 146 & & Cinncassiol $\mathrm{D}_{1}$ glucoside & $\mathrm{C}_{20} \mathrm{H}_{32} \mathrm{O}_{5}$ & 352 & {$[64,65]$} \\
\hline 147 & & 1-Hydroxycinncassiol $\mathrm{D}_{1}$ & $\mathrm{C}_{20} \mathrm{H}_{32} \mathrm{O}_{6}$ & 368 & {$[45]$} \\
\hline 148 & & Cinncassiol $\mathrm{D}_{2}$ & $\mathrm{C}_{20} \mathrm{H}_{32} \mathrm{O}_{6}$ & 368 & {$[64]$} \\
\hline 149 & & Cinncassiol $\mathrm{D}_{2}$ glucoside & $\mathrm{C}_{26} \mathrm{H}_{42} \mathrm{O}_{11}$ & 531 & {$[64]$} \\
\hline 150 & & Cinncassiol $\mathrm{D}_{3}$ & $\mathrm{C}_{20} \mathrm{H}_{32} \mathrm{O}_{5}$ & 352 & {$[64]$} \\
\hline 151 & & Cinncassiol $\mathrm{D}_{3}$-2-O-monoacetate & $\mathrm{C}_{24} \mathrm{H}_{36} \mathrm{O}_{8}$ & 452 & {$[46]$} \\
\hline 152 & & (18S)-3-Dehydroxycinncassiol $\mathrm{D}_{3}$ & $\mathrm{C}_{20} \mathrm{H}_{32} \mathrm{O}_{5}$ & 352 & {$[45]$} \\
\hline 153 & & $\begin{array}{l}\text { (18S)-3-Dehydroxycinncassiol } \\
\qquad \mathrm{D}_{3} \text { glucoside }\end{array}$ & $\mathrm{C}_{26} \mathrm{H}_{42} \mathrm{O}_{10}$ & 515 & {$[45]$} \\
\hline 154 & & $\begin{array}{l}\text { (18S)-3,5-Didehydroxy-1,8-dihydroxy- } \\
\text { cinncassiol } \mathrm{D}_{3}\end{array}$ & $\mathrm{C}_{20} \mathrm{H}_{32} \mathrm{O}_{6}$ & 368 & {$[45]$} \\
\hline 155 & & $\begin{array}{c}\text { (18S)-3-Dehydroxy-8-hydroxy } \\
\text {-cinncassiol } \mathrm{D}_{3}\end{array}$ & $\mathrm{C}_{20} \mathrm{H}_{32} \mathrm{O}_{6}$ & 368 & {$[45]$} \\
\hline 156 & & Cinncassiol $\mathrm{D}_{4}$ & $\mathrm{C}_{20} \mathrm{H}_{32} \mathrm{O}_{5}$ & 352 & {$[66]$} \\
\hline 157 & & Cinncassiol $\mathrm{D}_{4}$ glucoside & $\mathrm{C}_{26} \mathrm{H}_{42} \mathrm{O}_{10}$ & 515 & {$[67]$} \\
\hline 158 & & Cinncassiol $\mathrm{D}_{4}-2-\mathrm{O}$-monoacetate & $\mathrm{C}_{22} \mathrm{H}_{34} \mathrm{O}_{6}$ & 395 & {$[46]$} \\
\hline 159 & & 18-Hydroxyperseanol & $\mathrm{C}_{20} \mathrm{H}_{32} \mathrm{O}_{8}$ & 400 & {$[67,68]$} \\
\hline 160 & & Perseanol & $\mathrm{C}_{20} \mathrm{H}_{32} \mathrm{O}_{7}$ & 384 & {$[67,68]$} \\
\hline 161 & & $16-O-\beta$-D-Glucopyranosyl-perseanol & $\mathrm{C}_{26} \mathrm{H}_{42} \mathrm{O}_{13}$ & 563 & {$[45]$} \\
\hline 162 & & $\begin{array}{c}\text { 19-Dehydroxy-13-hydroxy } \\
\text {-cinncassiol } \mathrm{D}_{1}\end{array}$ & $\mathrm{C}_{20} \mathrm{H}_{32} \mathrm{O}_{6}$ & 368 & {$[45]$} \\
\hline 163 & & $\begin{array}{l}\text { (E)-3-Dehydroxy-13(18)-ene-19-O- } \beta \text {-D } \\
\text {-glucopyranosyl-cinncassia } \mathrm{D}_{3}\end{array}$ & $\mathrm{C}_{26} \mathrm{H}_{40} \mathrm{O}_{10}$ & 513 & {$[45]$} \\
\hline 164 & Cinncassiol E & Cinncassiol E & $\mathrm{C}_{20} \mathrm{H}_{30} \mathrm{O}_{8}$ & 398 & [67] \\
\hline 165 & Cinncassiol F & Cinncassiol F & $\mathrm{C}_{20} \mathrm{H}_{30} \mathrm{O}_{8}$ & 398 & {$[67]$} \\
\hline 166 & \multirow{7}{*}{ Cinncassiol G } & Cinncassiol G & $\mathrm{C}_{20} \mathrm{H}_{30} \mathrm{O}_{7}$ & 382 & [67] \\
\hline 167 & & 16-O- $\beta$-D-Glucopyranosyl-19-deoxycinncassiol G & $\mathrm{C}_{26} \mathrm{H}_{40} \mathrm{O}_{12}$ & 545 & {$[67]$} \\
\hline 168 & & Cinnamomol C & $\mathrm{C}_{20} \mathrm{H}_{30} \mathrm{O}_{6}$ & 366 & {$[45]$} \\
\hline 169 & & Cinnamomol D & $\mathrm{C}_{20} \mathrm{H}_{30} \mathrm{O}_{5}$ & 350 & {$[45]$} \\
\hline 170 & & Cinnamomol E & $\mathrm{C}_{20} \mathrm{H}_{30} \mathrm{O}_{6}$ & 366 & {$[45]$} \\
\hline 171 & & Cinnamomol F & $\mathrm{C}_{17} \mathrm{H}_{24} \mathrm{O}_{4}$ & 292 & {$[45]$} \\
\hline 172 & & 13-O- $\beta$-D-Glucopyranosyl-cinnamomol F & $\mathrm{C}_{23} \mathrm{H}_{34} \mathrm{O}_{9}$ & 455 & {$[45]$} \\
\hline 173 & \multirow{2}{*}{ Cinnamomane } & Cinnamomol A & $\mathrm{C}_{20} \mathrm{H}_{30} \mathrm{O}_{8}$ & 398 & {$[68]$} \\
\hline 174 & & Cinnamomol B & $\mathrm{C}_{20} \mathrm{H}_{30} \mathrm{O}_{9}$ & 414 & {$[68]$} \\
\hline 175 & \multirow{2}{*}{ Cassiabudane } & Cassiabudanol A & $\mathrm{C}_{21} \mathrm{H}_{32} \mathrm{O}_{8}$ & 412 & [69] \\
\hline 176 & & Cassiabudanol B & $\mathrm{C}_{20} \mathrm{H}_{30} \mathrm{O}_{8}$ & 398 & [69] \\
\hline 177 & \multirow{3}{*}{ Others } & Cinnacasol & $\mathrm{C}_{20} \mathrm{H}_{30} \mathrm{O}_{7}$ & 382 & {$[70]$} \\
\hline 178 & & Cinnacaside & $\mathrm{C}_{26} \mathrm{H}_{40} \mathrm{O}_{12}$ & 545 & [70] \\
\hline 179 & & Phytol & $\mathrm{C}_{20} \mathrm{H}_{40} \mathrm{O}$ & 297 & {$[54,71]$} \\
\hline
\end{tabular}



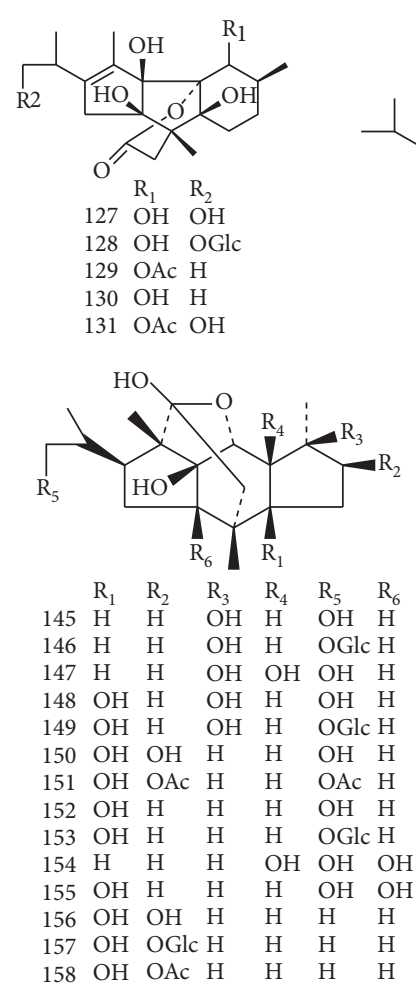

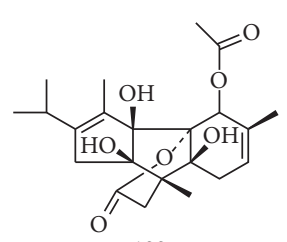

132

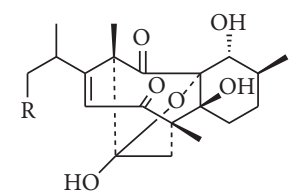

$141 \mathrm{R}=\mathrm{OH}$ $142 \mathrm{R}=$ OGlc $143 \mathrm{R}=\mathrm{H}$

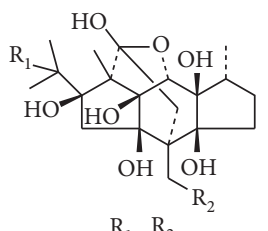

$\begin{array}{lll}\mathrm{R}_{1} & \mathrm{R}_{2}\end{array}$

$159 \mathrm{OH} \mathrm{H}$

$160 \mathrm{H} \quad \mathrm{H}$

$161 \mathrm{H}$ OGlc

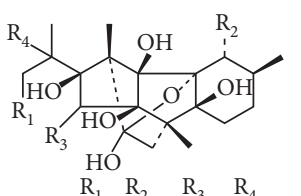

$\begin{array}{llll}\mathrm{R}_{1} & \mathrm{R}_{2} & \mathrm{R}_{3} & \mathrm{R}\end{array}$

$\begin{array}{llllll}134 & \mathrm{H} & \mathrm{OH} & \mathrm{H} & \mathrm{H}\end{array}$

$135 \mathrm{H}$ OAc $\mathrm{H} \quad \mathrm{H}$

$136 \mathrm{OH} \mathrm{OH} \mathrm{H} \quad \mathrm{H}$

$137 \mathrm{H} \quad \mathrm{OH} \quad \mathrm{OH} \mathrm{H}$

$138 \mathrm{H} \quad \mathrm{OH} \quad \mathrm{OAc} \mathrm{H}$

$139 \mathrm{H}$ OAc $\mathrm{H} \quad \mathrm{OH}$

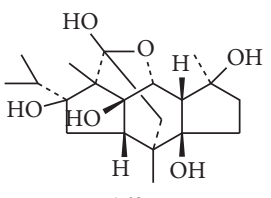

162
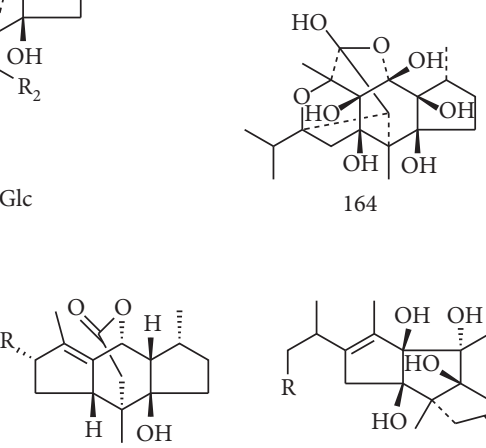

$171 \mathrm{R}=\mathrm{OH}$ $172 \mathrm{R}=$ OGlc

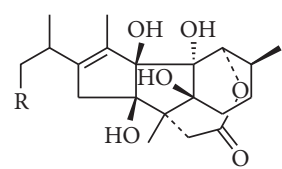

$173 \mathrm{R}=\mathrm{OH}$ $174 \mathrm{R}=$ OGlc

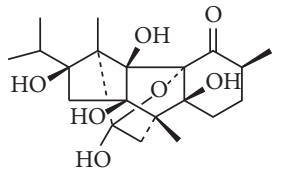

140

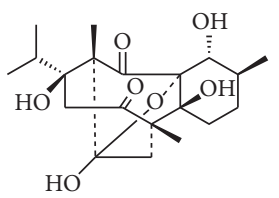

144
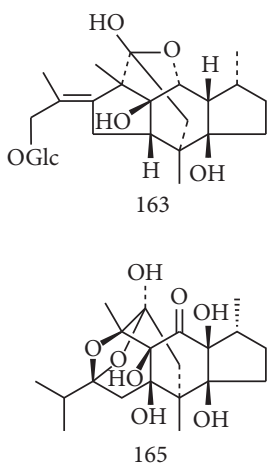

165

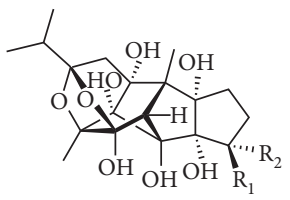

$\begin{array}{lll}\mathrm{R}_{1} & \mathrm{R}_{2}\end{array}$

$175 \mathrm{CH}_{3} \mathrm{H}$ $176 \mathrm{OH}^{\mathrm{CH}_{3}}$ $166 \mathrm{OH} \mathrm{H}^{\mathrm{R}_{1}} \mathrm{R}_{2}$ $167 \mathrm{H}$ OGlc

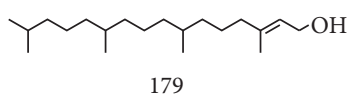

"- $\mathrm{OH}$

$177 \mathrm{R}=\mathrm{OCH}_{3}$

$178 \mathrm{R}=\mathrm{OH}$

Figure 3: The structures of diterpenes from Cinnamomum.
According to the research conducted by Zeng et al. [67], isoryanodane diterpenoid perseanol (160) is the plausible biosynthetic precursor of cinncassiol F (165) and cinncassiol G (166), and biosynthetic pathways have been proposed. These were, respectively, formed by the cleavage of the 12,13bond and 11,12-bond from $\mathbf{1 6 0}$ through a series of reactions under the catalysis of acid. In addition, Zhou et al. [68] proposed that cinnamomane diterpenoids are also generated from perseanol (160) through a series of retro-aldol, aldol, and oxidation reactions.

Some diterpenes of Cinnamomum have been reported to demonstrate immunomodulatory potency, covering immunostimulative and immunosuppressive activities.
Zhou et al. reported that the cassiabudane diterpenes 175 and 176 showed strong immunostimulative activity. Both of these significantly promoted the expansion of ConA-induced murine T cells with enhancement rates of 10.38 and $19.96 \%$, respectively, at a dose of $0.3906 \mu \mathrm{M}$ and enhanced the proliferation of LPS-induced murine B cells with enhancement rates of 52.13 and 55.06\%, respectively, at the same dose [69]. In another assay conducted by Zhou et al., cinnamomane diterpenes $\mathbf{1 7 3}$ and 174 could promote the proliferation of ConA-induced murine $\mathrm{T}$ cells with enhancement rates of 59 and $64 \%$, respectively, at the concentration of $0.3906 \mu \mathrm{M}$ [68]. By comparing the results, we found that cinnamomane 


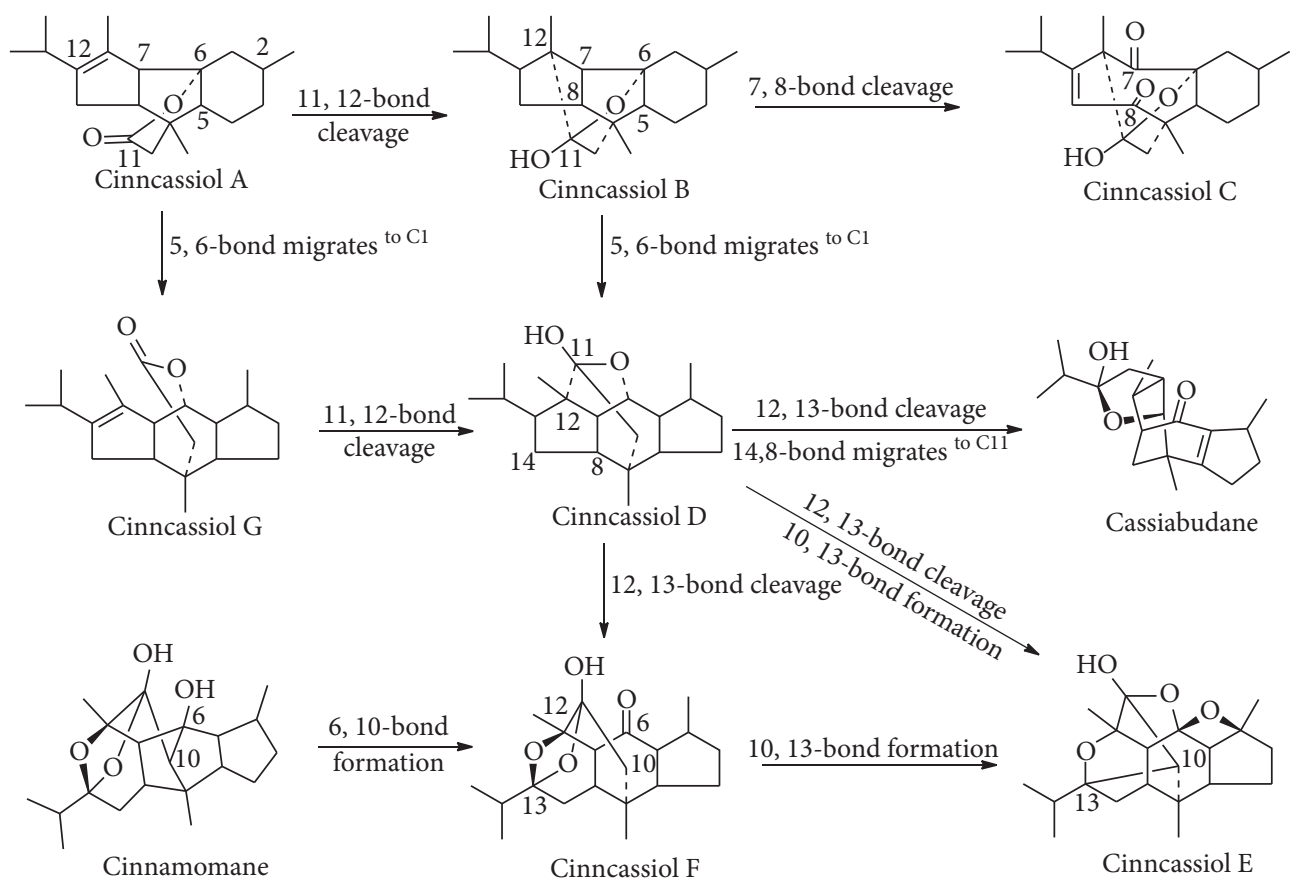

FIgURE 4: Biogenetic relationships of diterpene carbon skeletons from Cinnamomum.

tblBody

Table 4: Triterpenes from the genus Cinnamomum.

\begin{tabular}{lccccc}
\hline No. & Compound & Molecular formula & Molecular weight & Origin & References \\
\hline $\mathbf{1 8 0}$ & Squalene & $\mathrm{C}_{30} \mathrm{H}_{50}$ & 410 & $\mathrm{k}, \mathrm{q}, \mathrm{s}$ & {$[54,71]$} \\
$\mathbf{1 8 1}$ & Oleanolic acid & $\mathrm{C}_{30} \mathrm{H}_{48} \mathrm{O}_{3}$ & 456 & $\mathrm{~d}$ & {$[62]$} \\
\hline
\end{tabular}<smiles>CC(C)=CCC/C(C)=C/CC/C(C)=C/C/C=C(\C)CC/C=C(\C)CCC=C(C)C</smiles>

181

Figure 5: The structures of triterpenes from Cinnamomum.

diterpenes exhibited stronger immunostimulative effects than cassiabudane diterpenes, showing that the 6,10cyclo-12,13-seco-isoryanodane skeleton could probably promote the activity. In addition, a hydroxyl group connected to C-2 in cinnamomane diterpenes could enhance their immunostimulative activity and C-13 connecting to a hydroxyl group in cassiabudane diterpenes exerted stronger effect compared with connecting to a methoxy group.
In the ConA/LPS-induced splenocyte proliferation assay conducted by Zeng et al., cinncassiol G (166) inhibited $94.5 \%$ of ConA-induced murine T-cell proliferation at a dose of $100 \mu \mathrm{M}$, showing strong immunosuppressive activity. More importantly, 166 and 177 exhibited significant inhibitory effects on T-cell proliferation even at $50 \mu \mathrm{M}$, with inhibition rates of $86.1 \%$ and $58.8 \%$, respectively. It was also concluded that the 11,6 lactone moiety plays an important role in 
immunosuppressive activity, which accounts for the stronger inhibition of 166 compared to that of 177 [67]. According to the research by Zhou et al., some diterpenes, including $145,152,154,168-170,173$, and 174, could significantly promote ConA-induced murine T-cell proliferation, with proliferation rates of up to $78 \%$ at five different concentrations $(0.391$ to $100 \mu \mathrm{M})$. When the concentration was lower than $25 \mu \mathrm{M}$, compounds 168 and 169 appeared to promote proliferation. However, these appeared to inhibit the growth at the concentration of $100 \mu \mathrm{M}$ [45].

In addition, some diterpenes have been reported to exert anti-inflammatory activities. He et al. reported that compounds $127,129,130,133,134$, and 135 had a good performance of inhibiting $\mathrm{NO}$ production, with $\mathrm{IC}_{50}$ values ranging from 72.3 to $81.8 \mu \mathrm{M}$ [61].

2.4. Triterpenes. Two triterpenes were obtained from the Cinnamomum genus, including squalene (180) from $C$. kotoense, C. philippinense, and C. subavenium, and oleanolic acid (181) from C. camphora (Table 4 and Figure 5).

\section{Conclusion}

The medicinal value of the genus Cinnamomum has attracted much attention around the world, and many studies have been conducted. Most importantly, terpenes are the most abundant in the genus Cinnamomum, with various novel skeletons and significant biological activities, including immunomodulatory, anti-inflammatory, antimicrobial, antioxidant, and anticancer activities. In the present review, the research progress on chemical structures and biological effects of terpenes from Cinnamomum has been summarized. This can not only increase the understanding of the genus Cinnamomum, but also promote the better use of plants and further development of new drugs. Nevertheless, research on Cinnamomum remains incomplete, and more investigations are needed in the future.

First, chemical research on Cinnamomum plants has focused on only a few species like C. cassia and C. subavenium. Some other plants have gradually become research hotspots, but are only limited to the study of volatile oils. Cinnamomum plants have great economic and medicinal value and need to be further explored in future studies. Second, the diterpenes from Cinnamomum have shown significant immunomodulatory activities according to the findings. However, this only stays in the stage of cell activity in vitro. Thus, further pharmaceutical and in vivo activity experiments can be performed to promote the development of new drugs.

\section{Conflicts of Interest}

The authors declare that they have no conflicts of interest.

\section{Authors' Contributions}

M.T.W. conceptualized the review and drafted the initial version of the manuscript. L.N. and H.X.L. undertook the database search for the literature. H.Y.X., S.Q.Z., and X.X.Z. significantly contributed to the gathering of information and discussions of the manuscript. All authors read and approved the final manuscript.

\section{Acknowledgments}

This work was supported by the National Natural Science Foundation of China (no. 31700292) and Special Funds for Science and Technology Commissioners of Fujian Province (103/KTP19108A and 103/K1517070A).

\section{References}

[1] A. Asadollahi, M. Khoobdel, A. Z. Ramazani, S. Azarmi, and S. H. Mosawi, "Effectiveness of plant-based repellents against different Anopheles species: a systematic review," Malaria Journal, vol. 18, no. 1, p. 436, 2019.

[2] S. Kumar, R. Kumari, and S. Mishra, "Pharmacological properties and their medicinal uses of Cinnamomum: a review," Journal of Pharmacy and Pharmacology, vol. 71, no. 12, pp. 1735-1761, 2019.

[3] J. Zeng, Y. Xue, Y. Lai et al., "A new phenolic glycoside from the barks of Cinnamomum cassia," Molecules, vol. 19, no. 11, pp. 17727-17734, 2014.

[4] J. Zhao and J. S. Ma, "Phytochemicals and biological activities of the genus Cinnamomum," Journal of Pharmacognosy and Phytochemistry, vol. 4, no. 1, pp. 27-34, 2016.

[5] S. Wuu-Kuang, "Taxonomic revision of Cinnamomum (Lauraceae) in borneo," Blumea-Biodiversity, Evolution and Biogeography of Plants, vol. 56, no. 3, pp. 241-264, 2011.

[6] R. Singh and T. Jawaid, "Cinnamomum camphora (kapur): review," Pharmacognosy Journal, vol. 4, no. 28, pp. 1-5, 2012.

[7] D. F. Huang, J.-G. Xu, J.-X. Liu, H. Zhang, and Q. P. Hu, "Chemical constituents, antibacterial activity and mechanism of action of the essential oil from Cinnamomum cassia bark against four food-related bacteria," Microbiology, vol. 83, no. 4, pp. 357-365, 2014.

[8] X. Hao, J. Chen, Y. Lai et al., "Chemical constituents from leaves of Cinnamomum subavenium," Biochemical Systematics and Ecology, vol. 61, pp. 156-160, 2015.

[9] G. C. Huang, C. L. Kao, H. C. Yeh, W. J. Li, H. T. Li, and C. Y. Chen, "A new diphenyl ether from Cinnamomum subavenium," Chemistry of Natural Compounds, vol. 54, no. 5, pp. 869-871, 2018.

[10] A. M. Taha and O. A. Eldahshan, "Chemical characteristics, antimicrobial, and cytotoxic activities of the essential oil of Egyptian Cinnamomum glanduliferum bark," Chemistry and Biodiversity, vol. 14, no. 5, 2017.

[11] M. Saleem, H. N. Bhatti, M. I. Jilani, and M. A. Hanif, "Bioanalytical evaluation of Cinnamomum zeylanicum essential oil," Natural Product Research, vol. 29, no. 19, pp. 1857-1859, 2015.

[12] Y.-T. Tung, M.-T. Chua, S.-Y. Wang, and S.-T. Chang, "Antiinflammation activities of essential oil and its constituents from indigenous cinnamon (Cinnamomum osmophloeum) twigs," Bioresource Technology, vol. 99, no. 9, pp. 3908-3913, 2008.

[13] H. Jiang, J. Wang, L. Song et al., "GC $\times$ GC-TOFMS analysis of essential oils composition from leaves, twigs and seeds of Cinnamomum camphora L. Presl and their insecticidal and repellent activities," Molecules, vol. 21, no. 4, p. 423, 2016. 
[14] Z. Rao, F. Xu, T. Wen, F. Wang, W. Sang, and N. Zeng, "Protective effects of essential oils from Rimulus cinnamon on endotoxin poisoning mice," Biomedicine \& Pharmacotherapy, vol. 101, pp. 304-310, 2018.

[15] S.-G. Liao, T. Yuan, C. Zhang, S.-P. Yang, Y. Wu, and J.-M. Yue, "Cinnacassides A-E, five geranylphenylacetate glycosides from Cinnamomum cassia," Tetrahedron, vol. 65, no. 4 , pp. $883-887,2009$.

[16] X. Liu, J. Fu, X.-J. Yao et al., "Phenolic constituents isolated from the twigs of Cinnamomum cassia and their potential neuroprotective effects," Journal of Natural Products, vol. 81, no. 6, pp. 1333-1342, 2018.

[17] J.-F. Zeng, H.-C. Zhu, J.-W. Lu, L.-Z. Hu, J.-C. Song, and Y.-H. Zhang, "Two new geranylphenylacetate glycosides from the barks of Cinnamomum cassia," Natural Product Research, vol. 31, no. 15, pp. 1812-1818, 2017.

[18] H. Fuchino, A. Yazawa, F. Kiuchi, N. Kawahara, Y. Takahashi, and M. Satake, "Novel monoterpene lactones from Cinnamomum inunctum," Chemical and Pharmaceutical Bulletin, vol. 63, no. 10, pp. 833-836, 2015.

[19] Y. Guoruoluo, H. Zhou, W. Wang, J. Zhou, H. A. Aisa, and G. Yao, "Chemical constituents from the immature buds of Cinnamomum cassia (Lauraceae)," Biochemical Systematics and Ecology, vol. 78, pp. 102-105, 2018.

[20] Y.-Q. Li, D.-X. Kong, and H. Wu, "Analysis and evaluation of essential oil components of cinnamon barks using GC-MS and FTIR spectroscopy," Industrial Crops and Products, vol. 41, pp. 269-278, 2013.

[21] Y.-R. Li, C.-S. Fu, W.-J. Yang et al., "Investigation of constituents from Cinnamomum camphora (L.) J. Presl and evaluation of their anti-inflammatory properties in lipopolysaccharide-stimulated RAW 264.7 macrophages," Journal of Ethnopharmacology, vol. 221, pp. 37-47, 2018.

[22] M.-J. Cheng, W.-L. Lo, W.-S. Tseng, H.-C. Yeh, and C.-Y. Chen, "A novel normonoterpenoid from the stems of Cinnamomum reticulatum Hay," Natural Product Research, vol. 24, no. 8, pp. 732-736, 2010.

[23] R.-J. Lin, W.-L. Lo, Y.-D. Wang, and C.-Y. Chen, "A novel cytotoxic monoterpenoid from the leaves of Cinnamomum subavenium," Natural Product Research, vol. 22, no. 12, pp. 1055-1059, 2008.

[24] C.-H. Lee, C.-N. Kuo, H.-L. Chen, and C.-Y. Chen, "Review on pharmacological activities of Cinnamomum subavenium," Natural Product Research, vol. 27, no. 11, pp. 988-991, 2013.

[25] Z. E. Suntres, J. Coccimiglio, and M. Alipour, "The bioactivity and toxicological actions of carvacrol," Critical Reviews in Food Science and Nutrition, vol. 55, no. 3, pp. 304-318, 2015.

[26] I. A. Bnyan, A. T. Abid, and H. N. Obied, "Antibacterial activity of carvacrol against different types of bacteria," Journal of Natural Science Research, vol. 4, no. 9, pp. 13-16, 2014.

[27] M. Sharifi-Rad, E. M. Varoni, M. Iriti et al., "Carvacrol and human health: a comprehensive review," Phytotherapy Research, vol. 32, no. 9, pp. 1675-1687, 2018.

[28] A. Marchese, C. R. Arciola, R. Barbieri et al., "Update on monoterpenes as antimicrobial agents: a particular focus on p-cymene," Materials (Basel), vol. 10, no. 8, 2017.

[29] S.-S. Cheng, C.-G. Huang, Y.-J. Chen, J.-J. Yu, W.-J. Chen, and S.-T. Chang, "Chemical compositions and larvicidal activities of leaf essential oils from two eucalyptus species," Bioresource Technology, vol. 100, no. 1, pp. 452-456, 2009.

[30] E. Evergetis, A. Michaelakis, and S. A. Haroutounian, "Exploitation of Apiaceae family essential oils as potent biopesticides and rich source of phellandrenes," Industrial Crops and Products, vol. 41, pp. 365-370, 2013.

[31] A. S. Raeini, Z. Hafizibarjin, M. E. Rezvani, F. Safari, F. A. Aghda, and F. Z. Mehrjerdi, "Carvacrol suppresses learning and memory dysfunction and hippocampal damages caused by chronic cerebral hypoperfusion," NaunynSchmiedebergs Archives of Pharmacology, vol. 393, no. 4, pp. 581-589, 2020.

[32] Z. Hakimi, H. Salmani, N. Marefati et al., "Protective effects of carvacrol on brain tissue inflammation and oxidative stress as well as learning and memory in lipopolysaccharide-challenged rats," Neurotoxicity Research, vol. 37, no. 4, pp. 965976, 2020

[33] T. M. de Oliveira, R. B. F. de Carvalho, I. H. F. da Costa et al., "Evaluation of p-cymene, a natural antioxidant," Pharmaceutical Biology, vol. 53, no. 3, pp. 423-428, 2015.

[34] M. Hotta, R. Nakata, M. Katsukawa, K. Hori, S. Takahashi, and H. Inoue, "Carvacrol, a component of thyme oil, activates $\operatorname{PPAR} \alpha$ and $\gamma$ and suppresses COX-2 expression," Journal of Lipid Research, vol. 51, no. 1, pp. 132-139, 2010.

[35] W.-C. Lee, H. A. Jung, J. S. Choi, Y. S. Kim, and S.-M. Lee, "Protective effects of luteolin against apoptotic liver damage induced byd-galactosamine/lipopolysaccharide in mice," Journal of Natural Products, vol. 74, no. 9, pp. 1916-1921, 2011.

[36] J. Li, X. Zhang, and H. Huang, "Protective effect of linalool against lipopolysaccharide/D-galactosamine-induced liver injury in mice," International Immunopharmacology, vol. 23, no. 2, pp. 523-529, 2014.

[37] J. Ma, H. Xu, J. Wu, C. Qu, F. Sun, and S. Xu, "Linalool inhibits cigarette smoke-induced lung inflammation by inhibiting NF- $\kappa \mathrm{B}$ activation," International Immunopharmacology, vol. 29, no. 2, pp. 708-713, 2015.

[38] X. B. Sun, S. M. Wang, T. Li, and Y. Q. Yang, "Anticancer activity of linalool terpenoid: apoptosis induction and cell cycle arrest in prostate cancer cells," Tropical Journal of Pharmaceutical Research, vol. 14, no. 4, 2015.

[39] N. Okumura, H. Yoshida, Y. Nishimura, Y. Kitagishi, and S. Matsuda, "Terpinolene, a component of herbal sage, downregulates AKT1 expression in K562 cells," Oncology Letters, vol. 3, no. 2, pp. 321-324, 2012.

[40] M. G. de Oliveira, R. B. Marques, M. F. de Santana et al., "Alpha-terpineol reduces mechanical hypernociception and inflammatory response," Basic and Clinical Pharmacology and Toxicology, vol. 111, no. 2, pp. 120-125, 2012.

[41] A. T. Rufino, M. Ribeiro, F. Judas et al., "Anti-inflammatory and chondroprotective activity of $(+)-\alpha$-pinene: structural and enantiomeric selectivity," Journal of Natural Products, vol. 77, no. 2, pp. 264-269, 2014.

[42] P. Shu, X. Wei, Y. Xue et al., "Wilsonols A-L, megastigmane sesquiterpenoids from the leaves of Cinnamomum wilsonii," Journal of Natural Products, vol. 76, no. 7, pp. 1303-1312, 2013.

[43] X. Liu, J. Yang, J. Fu et al., "Phytochemical and chemotaxonomic studies on the twigs of Cinnamomum cassia (Lauraceae)," Biochemical Systematics and Ecology, vol. 81, pp. 45-48, 2018.

[44] A. Pardede, M. Adfa, A. Juliari Kusnanda, M. Ninomiya, and M. Koketsu, "Flavonoid rutinosides from Cinnamomum parthenoxylon leaves and their hepatoprotective and antioxidant activity," Medicinal Chemistry Research, vol. 26, no. 9, pp. 2074-2079, 2017.

[45] L. Zhou, "Studies on the Chemical Constituents and Immunomodulatory Activities of the Leaves of Cinnamomum 
Cassia", Huazhong University of Science and Technology, Wuhan, China, 2016.

[46] Q. L. Hu, "The Discovery of Components and Research on Bioactivities of Cinnamomum Cassia and Carpesium Abrotanoides", Lanzhou University, Lanzhou, China, 2018.

[47] C.-T. Chang, W.-L. Chang, J.-C. Hsu, Y. Shih, and S.-T. Chou, "Chemical composition and tyrosinase inhibitory activity of Cinnamomum cassia essential oil," Botanical Studies, vol. 54, no. 1, p. 10, 2013.

[48] Y.-M. Yan, P. Fang, M.-T. Yang, N. Li, Q. Lu, and Y.-X. Cheng, "Anti-diabetic nephropathy compounds from Cinnamomum cassia," Journal of Ethnopharmacology, vol. 165, pp. 141-147, 2015.

[49] C. Zhang, L. Fan, S. Fan et al., "Cinnamomum cassia presl: a review of its traditional uses, phytochemistry, pharmacology and toxicology," Molecules, vol. 24, no. 19, 2019.

[50] C.-Y. Chen and Y.-D. Wang, "Norcadinane sesquiterpene from the roots of Cinnamomum subavenium," Chemistry of Natural Compounds, vol. 47, no. 3, pp. 461-462, 2011.

[51] Y. Guoruoluo, H. Zhou, J. Zhou, H. Zhao, H. A. Aisa, and G. Yao, "Isolation and characterization of sesquiterpenoids from Cassia buds and their antimicrobial activities," Journal of Agricultural and Food Chemistry, vol. 65, no. 28, pp. 56145619, 2017.

[52] R.-J. Lin, M.-J. Cheng, J.-C. Huang et al., "Cytotoxic compounds from the stems of Cinnamomum tenuifolium," Journal of Natural Products, vol. 72, no. 10, pp. 1816-1824, 2009.

[53] Y. K. Xiong, X. L. Liu, Z. J. Mu et al., "Differences of chemical constituents of volatile oil from Cinnamomum before and after drying and comparison of anti-drug resistant bacteria activities," Research and Exploration in Laboratory, vol. 37, pp. 56-59, 2018.

[54] C. Y. Chen, Y. D. Wang, and C. J. Wang, "Chemical constituents from the leaves of Cinnamomum philippinense," Chemistry of Natural Compounds, vol. 46, no. 6, pp. 941-942, 2011.

[55] H.-C. Lin and S.-S. Lee, "Dibenzocycloheptanoids from the leaves of Cinnamomum subavenium," Journal of Natural Products, vol. 75, no. 10, pp. 1735-1743, 2012.

[56] S. Subehan, S. Kadota, and Y. Tezuka, "In VitroMechanismbased inactivation of cytochrome P450 3A4 by a new constituent of Cinnamomum burmani," Planta Medica, vol. 74, no. 12, pp. 1474-1480, 2008.

[57] C. Y. Chen and Y. D. Wang, "A novel sequiterpenoid from the leaves of Cinnamomum subavenium," Chemistry of Natural Compounds, vol. 47, no. 2, pp. 215-217, 2011.

[58] L. Hua-Yong, Z. Yu, M. Xiao-Pan, and J. Sai, "Novel sesquiterpenoids isolated from Chimonanthus praecox and their antibacterial activities," Chinese Journal of Natural Medicines, vol. 16, no. 8, pp. 621-627, 2018.

[59] V. C. Pham, T. T. A. Nguyen, T. O. Vu, T. Q. Cao, B. S. Min, and J. A. Kim, "Five new diterpenoids from the barks of Cinnamomum cassia (L.) J. Presl," Phytochemistry Letters, vol. 32, pp. 23-28, 2019.

[60] A. Yagi, N. Tokubuchi, T. Nohara, G. Nonaka, I. Nishioka, and A. Koda, "The constituents of cinnamomi cortex. I. Structures of cinncassiol A and its glucoside," Chemical \& Pharmaceutical Bulletin, vol. 28, no. 5, pp. 1432-1436, 1980.

[61] S. He, Y. Jiang, and P.-F. Tu, "Three new compounds from Cinnamomum cassia," Journal of Asian Natural Products Research, vol. 18, no. 2, pp. 134-140, 2016.

[62] T. Nohaka, I. Nishioka, N. Tokubuchi, K. Miyahara, and T. Kawasaki, "Cinncassiol $\mathrm{C}_{1}$, a novel type of diterpene from cinnamomi cortex," Chemical and Pharmaceutical Bulletin, vol. 28, no. 6, pp. 1969-1970, 1980.

[63] Y. Kashiwada, T. Nohara, T. Tomimatsu, and I. Nishioka, "Constituents of cinnamomi cortex. IV. Structures of cinncassiols $\mathrm{C}_{1}$ glucoside, $\mathrm{C}_{2}$ and $\mathrm{C}_{3}$," Chemical and Pharmaceutical Bulletin, vol. 29, no. 9, 1981.

[64] T. Nohara, Y. Kashiwada, K. Murakami et al., "Constituents of cinnamomi cortex. V. Structures of five novel diterpenes, cinncassiols D1, D1 glucoside, D2,D2 glucoside and D3," Chemical \& Pharmaceutical Bulletin, vol. 29, no. 9, pp. 2451-2459, 1981.

[65] T. Nohara, Y. Kashiwada, T. Tomimatsu, M. Kido, N. Tokubuchi, and I. Nishioka, "Cinncassiol D1 and its glucoside, novel pentacyclic diterpenes from cinnamomi cortex," Tetrahedron Letters, vol. 21, no. 27, pp. 2647-2648, 1980.

[66] T. Nohara, Y. Kashiwada, T. Tomimatsu, and I. Nishioka, "Two novel diterpenes from bark of Cinnamomum cassia," Phytochemistry, vol. 21, no. 8, pp. 2130-2132, 1982.

[67] J. Zeng, Y. Xue, P. Shu et al., "Diterpenoids with immunosuppressive activities from Cinnamomum cassia," Journal of Natural Products, vol. 77, no. 8, pp. 1948-1954, 2014.

[68] L. Zhou, Y. Tuo, Y. Hao et al., "Cinnamomols A and B, immunostimulative diterpenoids with a new carbon skeleton from the leaves of Cinnamomum cassia," Organic Letters, vol. 19, no. 11, pp. 3029-3032, 2017.

[69] H. Zhou, Y. Guoruoluo, Y. Tuo et al., "Cassiabudanols A and $\mathrm{B}$, immunostimulative diterpenoids with a Cassiabudane carbon skeleton featuring a 3-oxatetracyclo $\left[6 \cdot 6 \cdot 1 \cdot 0^{2,6} \cdot 0^{10,14}\right]$ pentadecane scaffold from Cassia buds," Organic Letters, vol. 21, no. 2, pp. 549-553, 2019.

[70] T. M. Ngoc, D. T. Ha, I.-S. Lee et al., "Two new diterpenes from the twigs of Cinnamomum cassia," Helvetica Chimica Acta, vol. 92, no. 10, pp. 2058-2062, 2009.

[71] F.-C. Chen, C.-F. Peng, I.-L. Tsai, and I.-S. Chen, "Antitubercular constituents from the stem wood of Cinnamomum kotoense," Journal of Natural Products, vol. 68, no. 9, pp. 1318-1323, 2005. 